\title{
Joint control of production, overhaul and preventive maintenance for a production system subject to quality and reliability deteriorations
}

\author{
HÉCTOR RIVERA-GOMEZ ${ }^{1}$, ALI GHARBI ${ }^{1}$, JEAN PIERRE KENNÉ ${ }^{2}$ \\ ${ }^{1}$ Automated Production Engineering Department, École de technologie supérieure, \\ Production System Design and Control Laboratory, Université du Québec \\ 1100 Notre Dame Street West, Montreal, QC, Canada, H3C 1K3 \\ hriver06@hotmail.com \\ ali.gharbi@etsmtl.ca \\ ${ }^{2}$ Mechanical Engineering Department, École de technologie supérieure, \\ Laboratory of Integrated Production Tehcnologies, Université du Québec \\ 1100 Notre Dame Street West, Montreal, QC, Canada, H3C 1K3 \\ jean-pierre.kenne@etsmtl.ca
}

\begin{abstract}
This research investigates the case of an unreliable manufacturing system subject to quality and reliability deterioration. In particular, we conjecture that the deterioration of the system leads to a continuous increase in the intensity of failures and a decrease on the quality of the parts produced. As such, deterioration implies a twofold effect on the manufacturing system. When the machine fails, minimal repair is conducted, leaving the machine at the same level of deterioration before failure. Hence, the quality of the parts produced and the failure intensity remain unchanged with this repair. Meanwhile, an overhaul refers to a perfect repair that completely restores the quality of the parts and the failure intensity of the machine. This option completely counters all the effects of the deterioration. Preventive maintenance may also be conducted, but it reduces the level of deterioration only partially, improving the quality of the units produced and the failure intensity just in part. These set of characteristics yield to the formulation of a new control model that simultaneously determines the optimal production plan, the overhaul and preventive maintenance strategies. Such a joint control policy minimizes the total cost including the inventory holding, backlog, overhaul, preventive maintenance and defectives costs over an infinite planning horizon. Since the dynamics of the system change as a function of the level of deterioration, it is necessary to use its history for a proper formulation; therefore a Semi-Markov decision process is used. Numerical methods are applied to determine the control policy and numerical examples are conducted as illustrations. An extensive sensitivity analysis is presented in order to confirm the structure of the control policy obtained and examine the effect of several parameters.
\end{abstract}


Keywords: Quality, manufacturing systems, optimal control, numerical methods, stochastic processes, preventive maintenance.

\section{Introduction}

Manufacturing system control is a very active domain that has been widely investigated during the years. However, today the influence of several phenomena on the optimal production policy is still not clear. For instance, the impact of quality aspects on the production policy requires more research. Additionally, if we consider the fact that in practice, manufacturing systems are subject to deterioration (caused by a number of factors including the environment, the accumulated wearing, usage, etc), then it is logical to expect that such deterioration is amenable, not only to reduce the normal system operation, but also may have somehow an impact on the quality of the parts produced. In this context, various types of maintenance activities are available to resolve the effects of deterioration, and precisely the determination of the optimal production plan and the most efficient maintenance strategies is the scope of this research.

A detailed overview of the literature reveals that the production control problem has been formulated as a stochastic optimal control model motivated mainly by the pioneering work of Kimemia and Gershwin (1983). They presented a hierarchical control algorithm for the production management of a failure-prone flexible manufacturing system. Later, based on their formulation, Akella and Kumar (1986) obtained an analytic solution for the case of one machine that produces only one part type. Most of the literature in this research area has its foundation on these works, and recent extensions address the production control problem from different perspectives. For example, Mok and Porter (2006) proposed a stochastic optimization procedure to estimate the production rate of a manufacturing system producing either single product-type or multiple product-types. Sajadi et al. (2008) proposed another production control model, where they determined the production rate of a network of multiple machines, and multiple products with restrictions of the feeding materials. In another study on production control, Gharbi et al. (2011) treated the case of an unreliable central machine where a reserve machine is called upon in support to response to the demand. Despite the relevance and diversity of these papers, a common characteristic is that the aspect of quality is disregarded. Undoubtedly, this is a major limitation, since real manufacturing systems may be disrupted by the existence of defective products.

Some ideas have been proposed over the years to analyze the impact of quality aspects on production systems. For instance, a detailed discussion about the inter-relation between quality and productivity was presented by Inman et al. (2003). In this work they identified relevant industrial research opportunities on the interaction of quality and production system design. In addition, an analytical model was proposed by Tempelmeier and Bürger (2000), where they analyzed the performance of flow production systems with imperfect production. Nevertheless, quality issues have in particular attracted much attention, following the series of works by Kim and Gershwin (2005, 2008) who introduced mathematical models to analyze the performance of manufacturing system also producing defective products. Their approach has been extended by Colledani and Tolio (2009, 2011) who developed mathematical methods to model manufacturing and 
inspection machines monitored by control charts. Even though these works are relevant, they are unfortunately focused on the determination of performance measures, and they have not paid attention to the control policy that governs the production system. A growing research area intends to deal with this matter of incorporating quality aspects in the production policy, as in Mhada et al. (2011), who studied the production control problem with defective products. They derived analytical expressions for the production threshold and for the optimal cost. Hajji et al. (2012) presented another application that includes defectives. They tackled the joint production control and product specification for an unreliable multiple-parts production system. These contributions notwithstanding, they assumed that the rate of defectives is constant during the whole lifetime of the machine. This is a restrictive assumption in real production. It is clear that production systems experience wear, usage, corrosion, etc, leading to progressive deterioration that may have severe effects on different aspects of the machine, such as quality, reliability, safety etc. Thus, the domain of deteriorating systems provides a useful framework for our research.

Generally, two kinds of approaches to the modeling of variations in the machine's conditions are commonly found in the existing literature on deteriorating systems. In the first approach, some authors, such as Lam (2004), use the number of failures as an indicator of the level of deterioration. In particular, he introduced a lifetime distribution that takes into account the effect of maintenance activities for a deteriorating system whose operating time after repair decreases. In another study, $\mathrm{Wu}$ and Clements-Croome (2006) succeeded to model complicated changing failure intensities, with the advantage of capturing the whole system's lifetime. The second approach utilizes the age of the machine as state feedback of the deterioration, for example, Lai and Chen (2005) studied the replacement policy of a two-unit system. The unit's failure rate increases with their age, and a failure rate interaction between units is also presented. Even combinations of both approaches are possible, as in Deyahem et al. (2011), who proposed a joint production and repair-replacement model, where the failure rate of the machine increases with its age, while the repair time increases with the number of failures. It is evident that the aforementioned papers addressed deterioration regardless of quality. By contrast, we conjecture in this research that deterioration may influence the quality of the parts produced. Considering the link with quality, we extend the concept of deterioration to study its effects on the control policy in further detail through age-based maintenance strategies.

In practice, preventive maintenance is widely applied in a number of industrial sectors to counter the effect of random failures and the loss of production. The effective implementation of preventive maintenance policies has been extensively studied as in Yulan et al. (2008) where the joint determination of preventive maintenance and production planning was presented. They considered an objective function, consisted of multiple objectives instead of a single objective as most of the literature. Furthermore Radhoui et al. (2010) develop a model for one machine system producing lots of product. In this model, they utilize the proportion of non-conforming units as decision variable to determine when to perform preventive maintenance. Also they define the size of the buffer stock. On their part, Dhouib et al. (2012) proposed a model that determines the production control and the age-based preventive maintenance policy that reduces the shift 
rate to the out of control state, where the system produces defective products. Recently, Rivera-Gómez et al. (2013) treated a deteriorating system where the quality of the parts decreases by the influence of the natural wear of the machine and imperfect repairs. In their model they defined an aging process through a set of operational states. In addition, they succeeded to determine the optimal production and repairs policies. As we can notice in the research works mentioned above, a few applications have dealt with preventive maintenance strategies for manufacturing systems producing defectives products. Nevertheless, a key observation is that none of them have considered the simultaneous effect of deterioration on the quality of the parts produced and the reliability of the system, and how their repercussions can be mitigated through preventive maintenance strategies. To an extent, our model addresses this drawback, extending previous models and assumptions.

It is particular worthwhile to mention that this research aims to deepen previous contributions (i.e., Radhoui et al. (2010), Deyahem et al. (2011)) presented in the literature review, by specifically seeking to introduce an integrated model that determines optimal strategies for a manufacturing system subjected to a combined effect of deterioration. In fact, the assertion is that the machine is severely affected by the twofold effect of deterioration, and the objective is to provide a wide overview of such effects that increase the failure intensity, and decrease the quality of the parts produced, something that has not yet been studied in the literature. Since the machine dynamics are affected by deterioration, we use the age of the machine to denote its history. Markovian models are therefore, not appropriate; instead we formulate a Semi-Markov model. The deterioration decisions considered imply two types of maintenance; a major overhaul, which completely restores the machine, and preventive maintenance that partially renews the level of deterioration. The problem yields to the simultaneous determination of the production, preventive maintenance and overhaul strategies that minimize the total incurred cost over an infinite planning horizon, comprising the inventory, backlog, overhaul, preventive maintenance and defectives cost. An extensive sensitivity analysis is also conducted to illustrate the robustness and effectiveness of the proposed control policy.

The remainder of the paper is organized as follows. In section 2, the notations and system description are defined. The control problem formulation is presented in Section 3. The numerical techniques applied are detailed in Section 4. A numerical example is illustrated in Section 5, and a sensitivity analysis is conducted in Section 6 to illustrate the usefulness of the obtained control policy. Discussions are given in Section 7 and the paper is finally concluded in Section 8.

\section{Notations and manufacturing system description}

In this section, we introduce the notations used in this paper, and describe the manufacturing system under consideration.

\subsection{Notations}


The following defines the symbols and notations used in the present research:

$\begin{array}{ll}x(t) & \text { Inventory level at time } t \\ a(t) & \text { Age of the machine at time } t \\ u(t) & \text { Production rate of the manufacturing system at time } t \\ d & \text { Demand rate } \\ \xi(t) & \text { Mode of the machine at time } t \\ \mathrm{Q}(\cdot) & \text { Transition rate matrix } \\ q_{\alpha \alpha^{\prime}}(\cdot) & \text { Transition rate form mode } \alpha \text { to mode } \alpha \\ \tau & \text { Jump time of } \xi(t) \\ \beta(\cdot) & \text { Rate of defectives } \\ \rho & \text { Discount rate } \\ \gamma^{\xi(t)}(\cdot) & \text { Cost rate function } \\ h(\cdot) & \text { Inventory/backlog cost function } \\ J(\cdot) & \text { Expected discounted cost function } \\ v(\cdot) & \text { Value function } \\ c^{+} & \text {Incurred cost per unit of produced parts for positive inventory } \\ c^{-} & \text {Incurred cost per unit of produced parts for backlog } \\ c_{r} & \text { Constant repair cost } \\ c_{o} & \text { Overhaul cost } \\ c_{p m} & \text { Preventive maintenance cost } \\ c_{d} & \text { Cost of defectives } \\ u_{\max } & \text { Maximum production rate of the manufacturing system } \\ \omega_{p}(\cdot) & \text { Control variable for the preventive maintenance } \\ \omega_{\overline{\mathrm{p}}}, \omega_{\mathrm{p}} & \text { Maximal and minimal preventive maintenance rate } \\ \omega_{o}(\cdot) & \text { Control variable for the major overhaul } \\ \omega_{\overline{\mathrm{o}}}, \omega_{\underline{\mathrm{o}}} & \text { Maximal and minimal overhaul rate } \\ \pi_{i} & \text { Limiting probability at mode } i \\ \theta_{\mathrm{f}} & \text { Adjustment parameter for the failure rate } \\ \theta_{\mathrm{d}} & \text { Adjustment parameter for the rate of defectives }\end{array}$

\subsection{Manufacturing system description}

We shall begin by stating that the manufacturing system analyzed in this paper concerns the case of a single machine producing one part-type. The machine can produce parts at different rates to satisfy a constant demand for products. These different rate options comprise the production decisions of the control policy. The main issue is, however, that the machine undergoes progressive deterioration which severely degrades its reliability and the quality of the parts produced. This is clearly an undesirable feature. Another significant consideration is that a couple of maintenance activities are available to mitigate the effects of deterioration. A major overhaul consists of a time-consuming and expensive repair that completely restores the machine, mainly its reliability and quality of parts, to initial conditions. Meanwhile preventive maintenance is less expensive, but it only makes a partial restoration of the level of deterioration of the machine. The demand for products is satisfied exclusively by conforming parts, and so is important to maintain 
a low rate of defectives to reduce the inherent defectives cost. Figure 1 illustrates the set of characteristics considered in our model. To make the description complete the objective of the model is to determine, simultaneously, the optimal production plan and the preventive maintenance and overhaul strategies that minimize the total cost. In this case the incurred total cost consists of the inventory, backlog, preventive maintenance, overhaul, repair and defectives costs.

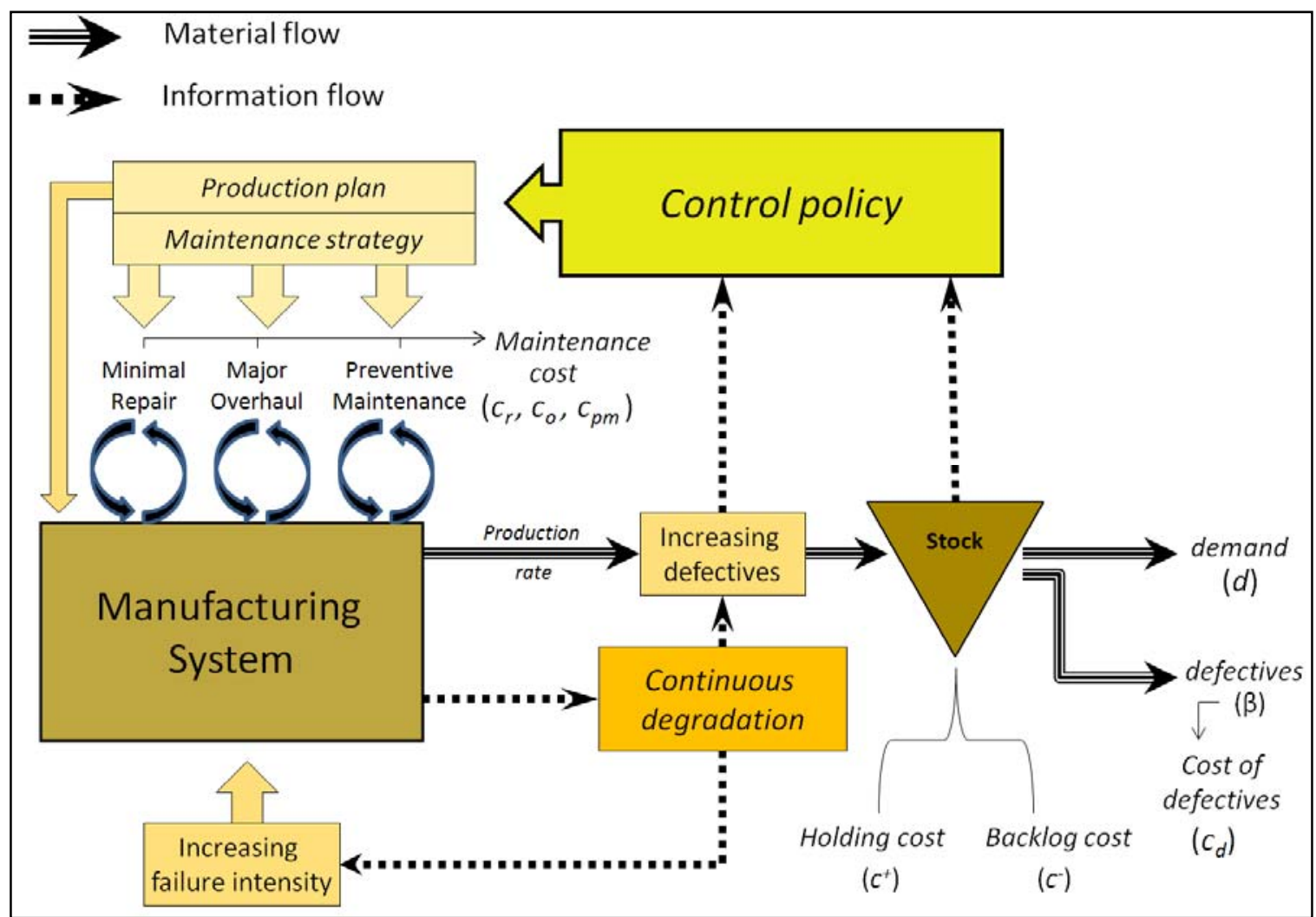

Figure 1: Manufacturing system considered

\section{Control problem formulation}

In the following subsections, we will describe the problem statement, the deterioration expressions and optimality conditions for our manufacturing system.

\subsection{Problem statement}

The aim of this subsection is to develop a stochastic dynamic programming model based on the optimal control theory that considers a twofold effect of deterioration as previously mentioned in section 2 . The system is subject to a number of random events (failures and repairs) and a couple of controlled actions (overhaul and preventive 
maintenance), thus this defines four different modes denoted by the random variable $\{\xi(t), t \geq 0\}$. The machine's modes can be classified as operational $\xi(t)=1$, at failure $\xi(t)=2$, under overhaul $\xi(t)=3$, and under preventive maintenance $\xi(t)=4$. Evidently, the mode of the machine at time $t$ denotes a continuous-time discrete state stochastic process $\xi(t) \in \Omega=\{1,2,3,4\}$ such that:

$$
\xi(t)=\left\{\begin{array}{cc}
1 & \text { operational } \\
2 & \text { failure } \\
3 & \text { overhaul } \\
4 & \text { preventive maintenance }
\end{array}\right.
$$

The machine may be at any of the four modes over an infinite horizon, as described in the following transition diagram:

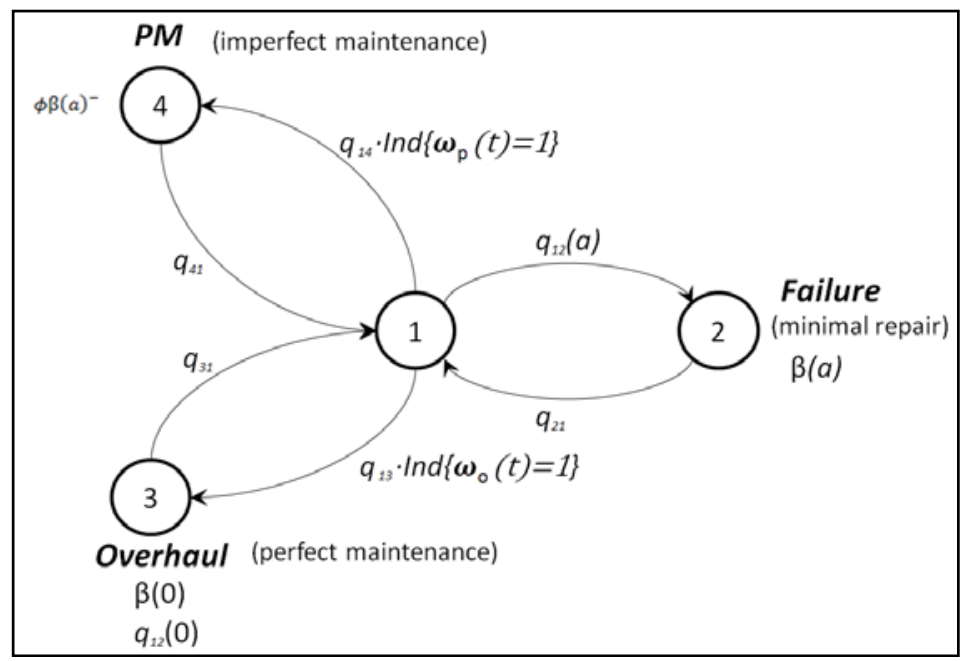

Figure 2: State transition diagram of the proposed model

Let $d$ be the constant demand rate to be satisfied, and $\mathrm{u}(t)$ the production rate at the time $t$, with $\mathrm{u}(t)=0$, if the machine is not operational (i.e., at failure, under overhaul or under preventive maintenance). When the machine is operational at time $t$, the production rate $\mathrm{u}(t)$ must satisfy the capacity constraint:

$$
0 \leq u(\cdot) \leq u_{\max }
$$

where $u_{\max }$ is the maximum production rate. To include the deterioration phenomenon in the model, in what follows we assume that deterioration has a twofold effect on the system, which can be observed on: a) the quality of the parts produced (implying an increasing rate of defectives $\beta$ ), and b) the reliability of the system (reducing the mean time to failure, MTTF, defined as the mean length of time that the machine is expected to last in operation). Thus, when the machine is operational, with $\xi(t)=1$, it produces a mix of conforming and defective products denoted by the rate of defectives $\beta$. However, when the machine is at failure, $\xi(t)=2$, the corrective maintenance conducted is not perfect, in fact a minimal repair that restores the system to as bad-as-old-conditions 
(ABAO) is carried out, where both $\beta$ and MTTF remain at the same values before repair. And when the machine is under overhaul, $\xi(t)=3$, a perfect maintenance that completely counters the effects of deterioration is performed, restoring the system (both $\beta$ and MTTF) to as-good-as-new conditions (AGAN), since the deterioration increases the rate of defectives and decreases the MTTF. Clearly these two maintenance options define two extreme cases between minimal and perfect repair. A more general option is when the system is under preventive maintenance, $\xi(t)=4$, since an imperfect maintenance is performed, restoring the system (mainly $\beta$ and MTTF) to somewhere between AGAN and ABAO conditions.

In modeling terms, our formulation stresses the fact that the process $\xi(t)$ is characterized by transition rates $q_{\alpha \alpha^{\prime}}(\cdot), \alpha, \alpha^{\prime} \in \Omega$, and due to deterioration, the time that the system remains operational decreases progressively, ultimately resulting in a Semi-Markov model. For this reason we need to employ the machine's history to determine appropriate control strategies. The history is denoted by the age of the machine $\mathrm{a}(t)$, therefore the conditional probability that the Semi-Markov process will stay in the same state $\alpha$ or will make a transition to state $\alpha^{\prime}$ within the next $\delta t$ time units, incorporates a feedback on the age $\mathrm{a}(t)$, as presented in the following two conditions:

\section{Condition 1:}

$$
\begin{aligned}
P[\xi(t+\delta t)= & \alpha \mid \xi(t)=\alpha, \quad x(t)=x, \quad \mathrm{a}(t)=\mathrm{a}, \quad u(t)=u] \\
& =1+q_{\alpha \alpha}(\mathrm{x}, \mathrm{a}, \mathrm{u}) \delta t+o(x, \mathrm{a}, \delta t)
\end{aligned}
$$

Condition 2:

$$
\begin{aligned}
P[\xi(t+\delta t) & \left.=\alpha^{\prime} \mid \xi(t)=\alpha, \quad x(t)=x, \quad \mathrm{a}(t)=\mathrm{a}, \quad u(t)=u\right] \\
& =q_{\alpha \alpha^{\prime}}(\mathrm{x}, \mathrm{a}, \mathrm{u}) \delta t+o(x, \mathrm{a}, \delta t)
\end{aligned}
$$

with:

$$
\begin{array}{rr}
q_{\alpha \alpha}(\mathrm{x}, \mathrm{a}, \mathrm{u})=-\sum_{\alpha \neq \alpha^{\prime}} q_{\alpha \alpha^{\prime}}(\mathrm{x}, \mathrm{a}, \mathrm{u}) & \lim _{\delta t \rightarrow 0} \frac{0(x, \mathrm{a}, \delta t)}{\delta t}=0 \\
q_{\alpha \alpha^{\prime}}(\mathrm{x}, \mathrm{a}, \mathrm{u}) \geq 0, & \forall \alpha, \alpha^{\prime} \in \Omega: \alpha \neq \alpha^{\prime}
\end{array}
$$

In principle, we can improve the performance of the manufacturing system by the use of the decision variables $\omega_{o}(\cdot)$ and $\omega_{p}(\cdot)$, which allow us to control the transition to the major overhaul or preventive maintenance, respectively. To this end, the decision to send the machine to these maintenance activities is undertaken when the machine is operational. For instance, when we set the overhaul decision variable to $\left[\omega_{o}(t)=1\right]$, the reciprocal of $\left[\mathrm{q}_{13} \cdot\left\{\omega_{o}(t)=1\right\}\right]$ represents the expected delay time between the decision to perform the overhaul and the effective switch from the operation mode to the 
overhaul mode. To say it in other words, the reciprocal corresponds to the delay between the call of a technician and its arrival. Meanwhile the machine remains operational when $\left\{\omega_{o}(t)=0\right\}$. A similar delay is represented by the reciprocal of $\left[\mathrm{q}_{14} \cdot\left\{\omega_{p}(t)=1\right\}\right]$, when the machine is sent to preventive maintenance. In addition, it follows that $\omega_{o}(\cdot)$ and $\omega_{p}(\cdot)$ define two binary variables that synchronize properly the transitions to the maintenance options available (overhaul an preventive maintenance), as indicated in the following expressions:

$\omega_{o}(t)=\left\{\begin{array}{l}1, \text { if } \text { Overhaul is conducted } \\ 0, \quad \text { otherwise }\end{array}, \quad \omega_{p}(t)=\left\{\begin{array}{l}1, \text { if } P M \text { is conducted } \\ 0, \text { otherwise }\end{array}\right.\right.$

The transitions matrix of the Semi-Markov chain $\xi(t)$, is denoted by $Q(\cdot)=\left\{\mathrm{q}_{\alpha \alpha^{\prime}}(\cdot)\right\}$, and it depends on the decision variables $\omega_{o}(\cdot), \omega_{p}(\cdot)$, as follows:

$$
\begin{aligned}
& Q\left(\omega_{o}, \omega_{p}\right)= \\
& \qquad\left(\begin{array}{cccc}
q_{11} & q_{12}(\mathrm{a}) & q_{13} \cdot \operatorname{Ind}\left\{\omega_{o}(t)=1\right\} & q_{14} \cdot \operatorname{Ind}\left\{\omega_{p}(t)=1\right\} \\
q_{21} & q_{22} & 0 & 0 \\
q_{31} & 0 & q_{33} & 0 \\
q_{41} & 0 & 0 & q_{44}
\end{array}\right)
\end{aligned}
$$

with the indicator function defined as:

$$
\operatorname{Ind}\{\Xi(t)\}=\left\{\begin{array}{l}
1 \text { if } \Xi(t) \text { is true } \\
0 \text { otherwise }
\end{array}\right.
$$

where $\Xi(t)$ indicates a given proposition. It follows that the control policy of the model is defined by the control variables $u(\cdot), \omega_{o}(\cdot)$ and $\omega_{p}(\cdot)$. Hence, the set of the feasible control policies $\Gamma(\alpha)$ which includes $\left(u(\cdot), \omega_{o}(\cdot)\right.$ and $\left.\omega_{p}(\cdot)\right)$, depends on the stochastic process $\xi(t)$ and is given by:

$$
\begin{aligned}
\Gamma(\alpha)=\left\{\left(u(\mathrm{a}, \cdot), \omega_{o}(\mathrm{a}, \cdot), \omega_{p}(\mathrm{a}, \cdot)\right) \in \mathrm{R}^{3},\right. \\
\left.0 \leq u(\mathrm{a}, \cdot) \leq u_{\max }, \quad \omega_{o}(\mathrm{a}, \cdot) \in\{0,1\}, \quad \omega_{p}(\mathrm{a}, \cdot) \in\{0,1\}\right\}
\end{aligned}
$$

In the stochastic control setting, normally the dynamics for the stock variable $x(\cdot)$ disregards the existence of defective products, as presented in Gershwin (2002). 
Nevertheless, our emphasis for this dynamics is to include two distinctive characteristics: i) the existence of defective products, and ii) the influence of deterioration. Hence, in our model the system dynamics evolve based on the following differential equation:

$$
\dot{x}(t)=u(t)-d(1+\beta(a)), \quad x(0)=x_{0},
$$

with $\mathrm{x}_{0}$, as the given initial inventory level, where $x(t)>0$ represents a positive inventory, and $x(t) \leq 0$ denotes the backlog of products. While $\beta(a)$ represents the rate of defectives as function of the age of the machine $\mathrm{a}(t)$. From the previous expression (8), we notice that the impact of defective products is to increase the demand to a higher level, as the quality of the machine deteriorates, to ensure that the demand is satisfied with flawless product. Moreover we define the age of the machine at time $t$, as an increasing function of its production rate since its last restart, then the cumulative age $a(t)$ is the solution of the following differential equation:

$$
\dot{\mathrm{a}}(t)=k_{1} \cdot u(t), \quad \mathrm{a}(\mathrm{T})=0,
$$

where $k_{1}$ is a given positive constant, and $\mathrm{T}$ represents the last restart time of the machine. In the next section, we provide further details about the expressions that complement the deterioration modeling, in special we clarify how exactly the age of the machine is linked with the effects of deterioration (namely, the increase on the rate of the defectives $\beta$, and the decrease on the MTTF). In closing this section, we note that the mathematical form of the problem actually entails that at any given instant of time $t$, the system is characterized by the following state variables:

- The machine mode, $\xi(t)$

- The stock level, $x(t)$

- The age of the machine, $\mathrm{a}(t)$

Consequently, the interpretation of the information provided by the vector $(\xi(t), x(t), \mathrm{a}(t))$ is fundamental to optimally control the proposed manufacturing system.

\subsection{Deterioration modeling}

In formulating the problem, details of how we model the deterioration phenomenon must be shown. We therefore, concentrate now on the expressions applied in this respect. At first glance, it appears that productive systems are subject to deterioration because of several factors, including usage, wear, aging, and so forth. Some authors have dealt with this matter, for example, Love et al. (2000) proposed that the failure rate depends on the age of the machine, and so they used that age to determine repair activities that reset the 
failure rate of the system. In the same direction, Dehayem et al. (2011) suggested that the deterioration of the machine is denoted by its age and number of failures, and the effect of deterioration is reflected at increasing several transitions rates. These articles lead to the identification of an age-deterioration relationship, which serves to propose in this paper that the trajectory of the failure rate of the machine $q_{12}$, is described by an increasing function of its age $a(t)$, because of the link between the age of the machine and its deterioration, as indicated in the following expression:

$$
q_{12}(\mathrm{a})=q_{1}+q_{2}\left(1-e^{-k_{2} \cdot \theta_{\mathrm{f}} \cdot\left[\mathrm{a}(t)^{3}\right]}\right)
$$

with:

$$
0 \leq \theta_{\mathrm{f}} \leq 1
$$

the parameter $\theta_{\mathrm{f}}$ is used to adjust the trend of the failure rate, $q_{1}$ is the value of the transition $q_{12}$ at initial conditions, $q_{2}$ is the boundary considered in the deterioration, and $k_{2}$ is a given constant. Expressions such as Equation (10) denotes a machine age increasing failure rate, and have been used by Boukas and Haurie (1990), Kenne and Gharbi (1999), and Gharbi and Kenne (2005) to model manufacturing systems with decreasing reliability. The role of the parameter $\mathrm{a}(t)^{n}$ is to accelerate the variation of the increasing function. Moreover, we introduce the parameters $\theta_{\mathrm{f}}$ and $\theta_{\mathrm{d}}$ (in Expression 3.11) to provide with a different speed of deterioration to the failure rate, and to the rate of defectives. Therefore, these parameters serve us to adjust separately the effects of deterioration on the production system.

The inverse of such transition $q_{12}$ (a) denotes the $\operatorname{MTTF}(\mathrm{a})$ in function of the age a, indicating the effect of the age of the machine on more frequent failures. A practical concern is that we can vary the parameter $\theta_{\mathrm{f}}$ to adjust the trajectory of the failure rate to a specific system, as presented in Figure 3 a (where we use $q_{1}=0.01, q_{2}=0.16$ and $\left.k_{2}=15 \times 10^{-6}\right)$.

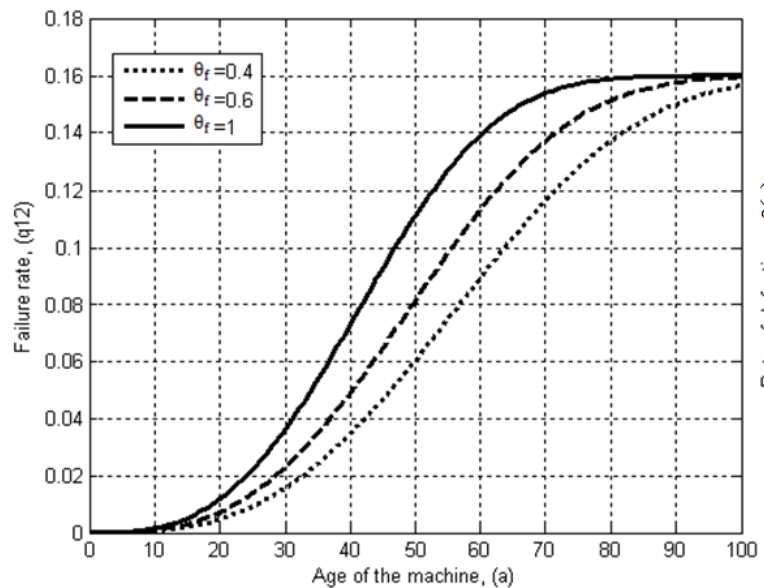

(a) For the failure rate, $q_{12}(a)$

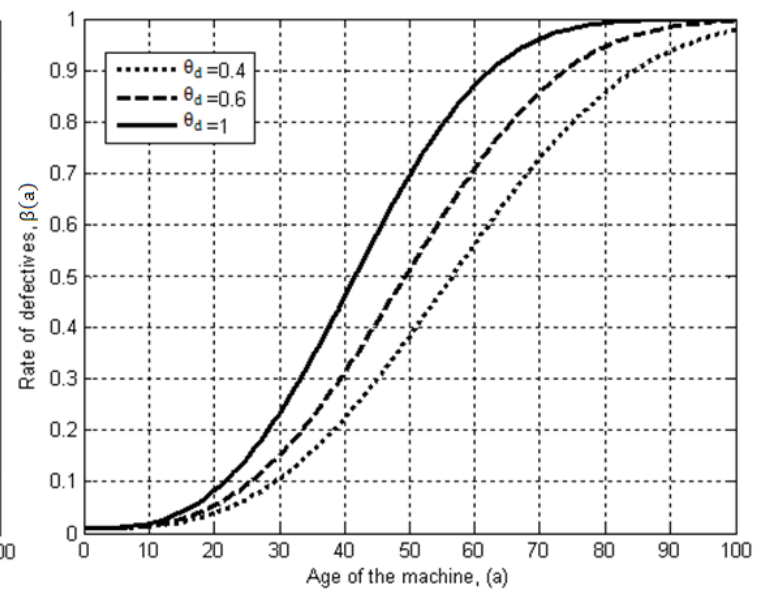

(b) For the rate of defectives, $\beta(a)$

Figure 3: Trend of deterioration 
Another important aspect is the fact that deterioration also has an effect on the quality of the parts produced. A number of authors have proposed the link between deterioration and quality, such as Kim and Gershwin (2008) who suggested that in modeling the wear of the machine, it can also be used to represent different quality yields. Additionally, Colledani and Tolio (2011) claimed that the existence of a degrading process in production systems may have a continuous deterioration on the quality of parts. These references permit to define an inter-relationship between deterioration and quality. In this way, based on the links of age-deterioration and deterioration-quality, claiming that in our model the rate of defectives $\beta$ can be modeled by an increasing function of the age of the machine $\mathrm{a}(t)$, is fairly straightforward. In this case we propose an increasing rate of defectives given by:

$$
\beta(\mathrm{a})=b_{1}+b_{2}\left(1-e^{-k_{3} \cdot \theta_{\mathrm{d}} \cdot\left[\mathrm{a}(t)^{3}\right]}\right)
$$

with:

$$
0 \leq \theta_{\mathrm{d}} \leq 1
$$

where $\theta_{\mathrm{d}}$ denotes the adjustment parameter for the trend of the rate of defectives as illustrated in Figure 3b, (with $b_{1}=0.01, b_{2}=0.99$ and $k_{3}=15 \times 10^{-6}$ ), $b_{1}$ is the value of the rate of defectives at initial conditions, $b_{2}$ is the boundary considered in the quality deterioration, and $k_{3}$ is a given constant. For the current problem, the parameters $\theta_{\mathrm{f}}$ and $\theta_{\mathrm{d}}$, captures one essential feature, namely, that their role is to emphasize whether the deterioration has a stronger effect on quality (rate $\beta$ ), or on the reliability (failure rate $q_{12}$ ). The analysis of maintenance service and quality data is the source to determine the value of the constants needed in Functions 10 and 11, and it can be useful, any historical information that indicate when the machine failed, how much time it was needed to repair the machine, and if it is observed a pattern of the deterioration that influence certain features of the machine. Additionally, well known increasing functions such as the Weibull distribution can be modeled by selecting suitable values for $k_{2}$ and $k_{3}$.

In regard to the preventive maintenance strategy, we state that it rejuvenates the age of the machine of an amount proportional to its age before preventive maintenance, in line with the so-called Arithmetic Reduction of Age (ARA) presented in Doyen and Gaudoin (2004). Hence, we propose the following expression to model the benefit of preventive maintenance:

$$
\mathrm{a}^{+}=\mathrm{a}^{-}-\phi_{p} \mathrm{a}^{-}
$$

where $\phi_{p}$ denotes the efficiency of preventive maintenance and satisfies the condition: $0<\phi_{p}<1$, whereas $\mathrm{a}^{-}$is the age of the machine before preventive maintenance, and $\mathrm{a}^{+}$is the age after preventive maintenance. In practical terms, expression (12) indicates that preventive maintenance reduces the wear out speed of the machine by an amount depending on the preventive maintenance efficiency $\phi_{p}$, and this reduction has a twofold effect on the failure rate and the rate of defectives. The benefit of preventive maintenance also can be altered with the parameters $\theta_{\mathrm{f}}$ and $\theta_{\mathrm{d}}$. An additional trait of expression (12) is that we can model different types of maintenance according to the value applied in the preventive maintenance efficiency $\phi_{p}$, as follows: 
- $\phi_{p}=1$ perfect maintenance, $q_{12}$ and $\beta$ are pulled back to AGAN conditions.

- $0<\phi_{p}<1$ imperfect maintenance, $q_{12}$ and $\beta$ reduce partially.

- $\phi_{p}=0 \quad$ minimal maintenance, $q_{12}$ and $\beta$ remain in ABAO conditions.

We proceed by presenting in Figure 4a, a possible reduction of the failure rate when the efficiency of preventive maintenance is set to $\phi_{p}=0.6$. This Figure 4 a enables to realize that preventive maintenance indeed provides a remarkable benefit. The effect for the rate of defectives is illustrated in Figure $4 \mathrm{~b}$. In general, both figures present the same behavior, reducing partially its deterioration level with every preventive maintenance. Even though, we use the same efficiency $\phi_{p}$ for both Figures 4 , we observe that the trajectories are not exactly the same; the differences observed are explained by the values of the parameters $\theta_{\mathrm{f}}, \theta_{\mathrm{d}}, k_{2}$ and $k_{3}$ applied in every figure.

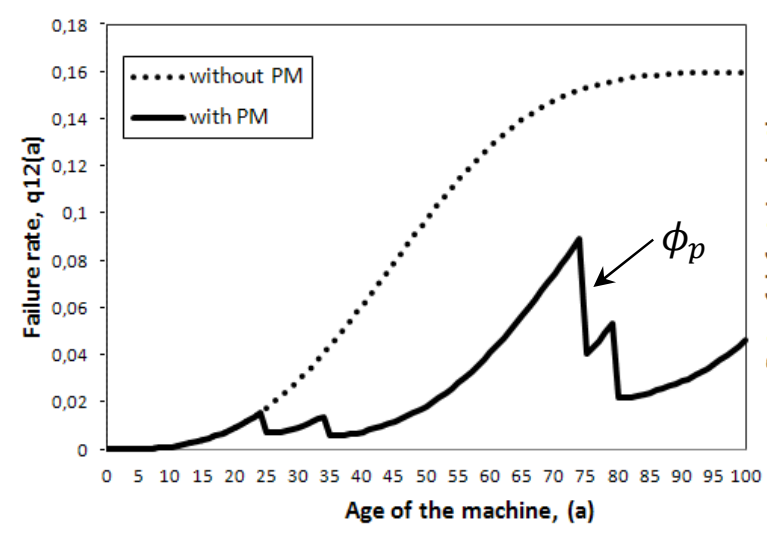

(a) On the failure rate, $q_{12}(a)$

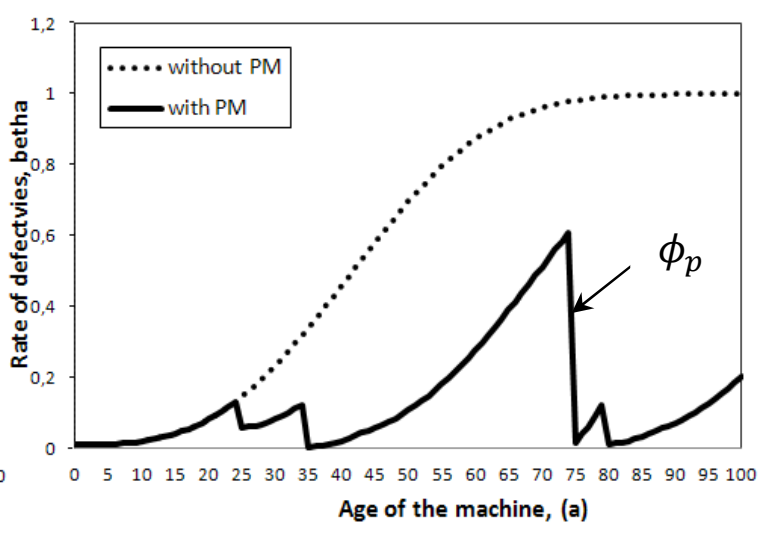

(b) On the rate of defectives, $\beta(a)$

Figure 4: Benefit of preventive maintenance

\subsection{Cost function and Optimality conditions}

For the sake of completeness, we will now formally declare that the state of the systems has a hybrid condition, formed by a discrete component $\xi(t)$, and continuous components, defined by $x(t)$ and $a(t)$, denoting the vector $(\xi(t), x(t), \mathrm{a}(t))$. The cost function $\gamma^{\xi(t)}(\cdot)$ of the model for each initial condition $(\xi(t), x(t), \mathrm{a}(t))$, is defined as follows:

$$
\begin{aligned}
& \gamma^{\xi(t)}(\alpha, x, \mathrm{a})= \\
& c^{+} x^{+}+c^{-} x^{-}+c_{r} \cdot \operatorname{Ind}\{\xi(t)=2\}+ \\
& \quad c_{o} \cdot \operatorname{Ind}\{\xi(t)=3\}+c_{p m} \cdot \operatorname{Ind}\{\xi(t)=4\}+c_{d} \cdot[\beta(\mathrm{a}) \cdot d]
\end{aligned}
$$

with:

$$
x^{+}=\max (0, x)
$$




$$
x^{-}=\max (-x, 0)
$$

where $c^{+}$and $c^{-}$are given constants used to penalize the inventory and backlog of parts, every time that the machine is sent to maintenance, it is incurred a cost depending of the maintenance option conducted. We denoted $c_{r}$ as the repair cost, $c_{o}$ is the overhaul cost, $c_{p m}$ is the preventive maintenance cost, and $c_{d}$ is the defectives cost which is originated by the additional inspection and handling costs related to defective products. The objective of our model is to determine a control policy that minimizes the integral of the following expected discounted cost:

$$
\begin{aligned}
& J(\alpha, x, \mathrm{a})= \\
& E\left[\int_{0}^{\infty} e^{-\rho t} \gamma^{\xi(t)}(\cdot) d t \mid \quad \xi(0)=\alpha, x(0)=x, \mathrm{a}(0)=\mathrm{a}\right], \quad \forall\left(u, \omega_{o}, \omega_{p}\right) \in \Gamma(\alpha)
\end{aligned}
$$

where $\rho$ is the discount rate, and $(\alpha, x, \mathrm{a})$ are the initial conditions of the state variables. The optimal decisions for this problem $\left(\mathrm{u}^{*}, \omega_{\mathrm{o}}^{*}, \omega_{\mathrm{p}}^{*}\right)$, minimizes $J(\cdot)$, and simultaneously defines the production, overhaul and preventive maintenance rates, as a function of the mode of the system, the inventory level and the age of the machine. The value function of the problem is defined as follows:

$$
\begin{aligned}
& v(\alpha, x, \mathrm{a})= \\
& \inf _{\left(\boldsymbol{u}, \omega_{o}, \omega_{P}\right) \in \Gamma(\alpha)} J\left(\alpha, x, \mathrm{a}, u, \omega_{o}, \omega_{p}\right), \quad \forall \alpha \in \Omega, x \in R, \mathrm{a} \in R
\end{aligned}
$$

The value function $v(\alpha, x, \mathrm{a})$ denotes the optimum value of the cost function (14) when the optimal control policy $\left(\mathrm{u}^{*}, \omega_{\mathrm{o}}^{*}, \omega_{\mathrm{p}}^{*}\right)$ is applied. One important point to note is that $v(\alpha, x, \mathrm{a})$ satisfies specific properties known as optimality conditions which can be derived regarding the principle of optimality. Let for instance $v(\cdot, t)$ denote a cost-to-go function at time $t$, assuming that we know the best possible control trajectory during the time interval $[t, \infty]$, but we know nothing in the interval $[0, t]$, hence we can break up Equation (15) into two parts as follows:

$v(\alpha(0), x(0), \mathrm{a}(0), 0)=$ 


$$
\inf _{\substack{u(t), \omega_{o}(t), \omega_{p}(t) \\ 0 \leq t \leq \infty}} E\left\{\int_{0}^{t} e^{-\rho t} \gamma^{\xi(t)}(\cdot) d t+\int_{t}^{\infty} e^{-\rho t} \gamma^{\xi(t)}(\cdot) d t \mid \alpha(0), x(0), \mathrm{a}(0)\right\}
$$

To handle the randomness of $\alpha$, the expectation operator $E$ is needed. We can considerably simplify Equation (16), noting that the second integral in the interval $[t, \infty]$ is the value function $v(\alpha(t), x(t), \mathrm{a}(t), t)$ and reducing the discounted rate. Moreover assuming that the value function is differentiable allows us to apply its full derivative. Therefore, after extensive manipulations, we have:

$$
\begin{aligned}
& \rho v(\alpha, x, \mathrm{a}, t)-\frac{\partial v}{\partial t}[\alpha, x, \mathrm{a}, t]= \\
& \inf _{u(t), \omega_{o}(t), \omega_{p}(t)}\left\{\gamma^{\alpha}\left[\alpha, x, \mathrm{a}, u, \omega_{o}, \omega_{p}\right]+\frac{\partial v}{\partial x}[\alpha, x, \mathrm{a}, t] \dot{x}+\frac{\partial v}{\partial \mathrm{a}}[\alpha, x, \mathrm{a}, t] \dot{a}+\sum_{\alpha^{\prime}} v\left[\alpha^{\prime}, x, \mathrm{a}, t\right] \lambda_{\alpha^{\prime} \alpha}\right\}
\end{aligned}
$$

To simplify matters, since the time horizon is infinite, and a steady-state distribution exists for $\alpha$, we can state that Equation (17) is independent of $t$, thus eliminating $t$ and $\frac{\partial v}{\partial t}$. By replacing the summation term by the generator $Q(\cdot)=\left\{q_{\alpha \alpha^{\prime}}(\cdot)\right\}$, finally Equation (17) is reduced to:

$$
\begin{aligned}
& \rho v(\alpha, x, \mathrm{a})= \\
& \min _{\left(u, \omega_{o}, \omega_{p}\right) \in \Gamma(\alpha)}\left\{\gamma^{\alpha}\left[\alpha, x, \mathrm{a}, u, \omega_{o}, \omega_{p}\right]+\frac{\partial v}{\partial x}[\alpha, x, \mathrm{a}] \dot{x}+\frac{\partial v}{\partial \mathrm{a}}[\alpha, x, \mathrm{a}] \dot{\mathrm{a}}+Q(\cdot) v[\alpha, x, \varphi(\xi, \mathrm{a})](\alpha)\right\}
\end{aligned}
$$

with:

$$
\varphi(\xi, \mathrm{a})=\left\{\begin{array}{ccc}
0 & \text { if } \xi\left(\tau^{+}\right)=1 & \text { and } \xi\left(\tau^{-}\right)=3 \\
\left(1-\phi_{p}\right) \mathrm{a}\left(\tau^{-}\right) & \text {if } \xi\left(\tau^{+}\right)=1 \quad \text { and } \xi\left(\tau^{-}\right)=4 \\
\mathrm{a}\left(\tau^{-}\right) & \text {otherwise }
\end{array}\right.
$$

where $\xi(t)=\alpha \in \Omega, \frac{\partial v}{\partial x}$ and $\frac{\partial v}{\partial \mathrm{a}}$ are the partial derivatives of the value function $v(\cdot) ; \tau$ denotes the first jump time of $\xi(t)$, and $\varphi(\xi, a)$ defines a reset function that describes the benefit of the maintenance activities available in the model. The relevance of Expression (18) stems from its capacity to define the fundamental manufacturing system control equations so-called Hamilton-Jacobi-Bellman (HJB) equations, which are essentially important because they lead to the optimal feedback control $\left(\mathrm{u}^{*}, \omega_{\mathrm{o}}^{*}, \omega_{\mathrm{p}}^{*}\right)$. Further details 
about the procedure to obtain HJB equations, can be consulted in Gershwin (2002). Nevertheless, the major inherent difficulty in HJB equations is that analytical solutions are cumbersome to obtain, instead numerical methods have been employed since Boukas and Haurie (1990). In the next section, we will look at the approach we adopted, in more detail.

\section{Numerical approach}

The present section provides further detail of the numerical method applied to solve the HJB equations (18) presented in section 3. The chief difficulty is to find an analytical solution of the HJB equations due to their complex structure which involves the solution of a coupled set of partial differential equations. We overcome such a difficulty by applying of a numerical method based on the Kushner technique. The main idea behind this approach is to use an approximation scheme for the gradient of the value function $v(\alpha, x, \mathrm{a})$, which replaces the unbounded domain of the continuous variables $\left(x\right.$, a) by a large but bounded domain, defined in a finite grid of discrete variables $G_{x a}$. Thus, the Kushner technique approximates the value function $v(\alpha, x, a)$ by a discrete function $v^{h}(\alpha, x, a)$, and the gradients $\frac{\partial v}{\partial x}$ and $\frac{\partial v}{\partial a}$ are approximated by:

$$
\frac{\partial v}{\partial x}(\alpha, x, \mathrm{a})= \begin{cases}\frac{1}{h_{x}}\left[v ^ { h } \left(\left(\alpha, x+h_{x}, \mathrm{a}\right)-v^{h}((\alpha, x, \mathrm{a})]\right.\right. & \text { if } \quad \dot{x} \geq 0 \\ \frac{1}{h_{x}}\left[v ^ { h } \left((\alpha, x, \mathrm{a})-v^{h}\left(\left(\alpha, x-h_{x}, \mathrm{a}\right)\right]\right.\right. & \text { if } \quad \dot{x}<0\end{cases}
$$

and

$$
\frac{\partial v}{\partial \mathrm{a}}(\alpha, x, \mathrm{a})=\frac{1}{h_{a}}\left[v^{h}\left(\left(\alpha, x, \mathrm{a}+h_{\mathrm{a}}\right)-v^{h}(\alpha, x, \mathrm{a})\right]\right.
$$

where $h_{x}$ and $h_{\mathrm{a}}$ denote the length of the finite differential interval of the variables $x$ and a, respectively. More details about the Kushner technique can be consulted in Kushner and Dupuis (1992) and Kenné et al. (2003). The HJB equation (18) can be expressed as a function of $v^{h}\left(\alpha, x\right.$, a) with step size $h_{x}$ and $h_{\mathrm{a}}$ on the grid $G_{x \mathrm{a}}$, and this implies discrete dynamic equations for every mode of the machine. Eventually, the solution of the discrete form of the HJB equations will tend to the value of $v^{h}(\alpha, x, a)$ as $h_{x}$ and $h_{\mathrm{a}}$ tends to zero, and successively, it will also provide the structure of the optimal control policy. In this case the discrete equation derived by the numerical approach is defined as follows:

$v^{h}(\alpha, x, \mathrm{a})=$ 


$$
\begin{gathered}
\min _{\left(u, \omega_{o}, \omega_{p}\right) \in \Gamma(\alpha)}\left[( \rho + | \mathrm { q } _ { \alpha \alpha } | + \frac { | \mathrm { r } | } { h _ { x } } + \frac { k _ { 1 } \cdot u } { h _ { \mathrm { a } } } ) ^ { - 1 } \left(\gamma^{\alpha}(\cdot)+v^{h}\left(\alpha, \mathrm{x}, \mathrm{a}+h_{\mathrm{a}}\right) \frac{k_{1} \cdot u}{h_{\mathrm{a}}}\right.\right. \\
+v^{h}\left(\alpha, x+h_{x}, \mathrm{a}\right) \frac{|r|}{h_{x}} \operatorname{Ind}\{\mathrm{r} \geq 0\}+v^{h}\left(\alpha, x-h_{x}, \mathrm{a}\right) \frac{|r|}{h_{x}} \operatorname{Ind}\{\mathrm{r}<0\} \\
\left.\left.+\sum_{\alpha \prime \neq \alpha} q_{\alpha \alpha^{\prime}}(\cdot) v\left(\alpha^{\prime}, x, \varphi(\xi, \mathrm{a})\right)\right)\right] \quad \forall \alpha \in \Omega, x \in R, \quad \mathrm{a} \in R
\end{gathered}
$$

where $\mathrm{r}=u(t)-d(1+\beta(\mathrm{a}))$. From a mathematical point of view, the discrete optimality conditions (21) obtained, replace the original infinite horizon problem by a discrete-time and discrete-state Semi-Markov decisions process that is much easier to solve. Actually, we use the policy improvement method to determine the optimal policy of the problem.

\section{Numerical example}

Through the following section, a numerical example is conducted as an illustration of the manufacturing system presented in section 2. As mentioned previously, the system capacity is described by a four state Semi-Markov process with mode in $\Omega=\{1,2,3,4\}$. The objective of the numerical example is to analyze the effectiveness of the simultaneous production, overhaul and preventive maintenance policies for a deteriorating system with feedback on the age of the machine and the stock level. We consider the discrete grid $G_{x a}$ that defines the computational domain for the inventory level $x$, and the age of the machine a, as follows:

$$
G_{x \mathrm{a}}=\{(\mathrm{x}, \mathrm{a}):-10 \leq \mathrm{x} \leq 20, \quad 0 \leq \mathrm{a} \leq 100\}
$$

We define the value of the required parameters of Equation (9) as: $k_{1}=0.1$, for Equation (10) as: $k_{2}=15 \times 10^{-6}, q_{1}=0.0001, q_{2}=0.16$ and for Equation (11) as: $k_{3}=15 \times 10^{-6}, b_{1}=0.01$ and $b_{2}=0.99$. Applying these values we can model a wide interval for the rate of defectives and the MTTF. This will facilitate the analysis of several scenarios, from AGAN conditions, until extreme scenarios with high levels of defective products and higher failures intensities. The transition rate from the failure mode to the operational mode is defined as constant with value $q_{21}=1$, and the transitions rates from overhaul and from preventive maintenance to the operational mode are also constant, with $q_{31}=3.5$ and $q_{41}=5$, respectively. Note that the transitions from the operational mode to overhaul or to preventive maintenance imply a shorter delay, with values: $q_{13}=12$ and $q_{14}=12$. In addition, we set $u_{\max }=14$, this parameter indicates the number of produced parts per unit of time, and $d=6$ denotes the demand of product per unit of time. One should remark that the manufacturing system satisfies the condition of feasibility: 


$$
u_{\max } \cdot \pi_{1}>d[1+\beta(\mathrm{a})]
$$

where $\pi_{1}$ denotes the limiting probability for the operational mode, which in this case is computed as follows:

$$
\pi_{1}=\frac{1}{1+\frac{\mathrm{q}_{12}}{\mathrm{q}_{21}}+\frac{\mathrm{q}_{13}}{\mathrm{q}_{31}}+\frac{\mathrm{q}_{14}}{\mathrm{q}_{41}}}
$$

It turns out that the feasibility condition guarantees the demand satisfaction of products even in the worse scenarios, which are observed when both $\beta$ and $q_{12}$ are at their peak.

\begin{tabular}{|c|c|c|c|c|c|}
\hline Parameter: & $\begin{array}{c}\mathrm{C}^{+} \\
\text {(\$/products/time units) }\end{array}$ & $\begin{array}{c}\text { C- } \\
\text { (\$/products/time units) }\end{array}$ & $\begin{array}{c}\mathrm{C}_{\mathrm{r}} \\
(\$ / \text { repair})\end{array}$ & $\begin{array}{c}\mathrm{C}_{\mathrm{O}} \\
\text { (\$/repair) }\end{array}$ & $\begin{array}{c}\mathrm{C}_{\mathrm{pm}} \\
(\$ / \mathrm{PM})\end{array}$ \\
\hline Value: & 11 & 150 & 7 & 10 & 5 \\
\hline Parameter: & $\begin{array}{c}\mathrm{C}_{\mathrm{d}} \\
\text { (\$/products) }\end{array}$ & $h_{x}$ & $h_{\mathrm{a}}$ & $\rho$ & $\phi_{p}$ \\
\hline Value: & 2 & 0.5 & 0.5 & 0.9 & 0.6 \\
\hline Parameter: & $\theta_{\mathrm{d}}$ & $\theta_{\mathrm{f}}$ & $\omega_{\underline{\mathrm{o}}}$ & $\omega_{\bar{o}}$ & $\underset{\text { (product/time units) }}{u_{\max }}$ \\
\hline Value: & 0.6 & 0.7 & 0 & 1 & 14 \\
\hline Parameter: & $\omega_{\underline{p}}$ & $\omega_{\bar{p}}$ & $\begin{array}{c}d \\
\text { (products/time units) }\end{array}$ & & \\
\hline Value: & 0 & 1 & 6 & & \\
\hline
\end{tabular}
The rest of the parameters needed in the numerical example are presented in Table 1.

Table 1. Parameters for the numerical example

The following results were obtained with the data presented in Table 1, and are analyzed throughout this section to clearly illustrate the structure of the joint production, overhaul and preventive maintenance policies that optimally control the manufacturing systems of interest.

\subsection{Production Policy}

We concentrate first on the optimal production policy $\mathrm{u}^{*}(\alpha, \mathrm{x}, \mathrm{a})$, which indicates the production rate for a certain stock level $x(t)$ and age of the machine $a(t)$. Based on the numerical results presented in Figure 5a, the production policy defines three control rules, where the production rate is set to $u_{\max }, d$ and 0 . More specifically these rules state that: a) If the stock level is inferior to the corresponding optimal stock level $Z_{p}^{*}(\cdot)$, then the production rate should be set to the maximum rate. b) Once the stock level is equal to the optimal threshold level, the production rate should be set to the demand rate. c) If the current stock level exceeds the optimal stock level, then the system does not produce at all, and the production rate is set to zero. 
From these rules, one can clearly observe an extra characteristic in Figure 5a, where the consequence of include deterioration in the model, has the impact of progressively increasing the production threshold. Specifically, we observe that as the age of the machine increases, it also increases the failure rate and the rate of defectives, then the production threshold increases to ensure the demand satisfaction with flawless products. We use the boundary of the optimal production policy presented in Figure 5b to better illustrate its pattern, which serves to more easily identify the optimal stock level $Z_{p}^{*}(\cdot)$. From this Figure 5b we identify two zones: The zone $A_{u}$, where the optimal production policy recommends producing at maximum rate to reach the hedging point $Z_{p}^{*}(\cdot)$, and the zone $B_{u}$, where the recommendation is to not produce at all.

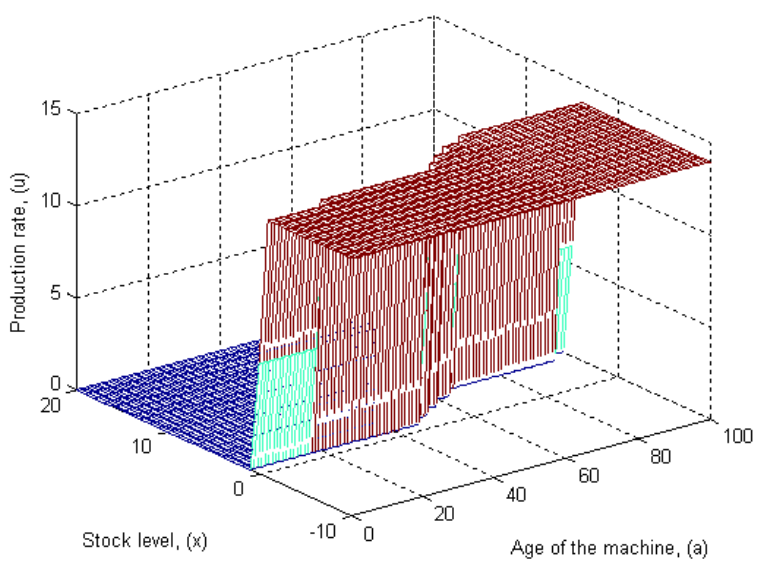

a) Production rate versus stock level and age of the machine

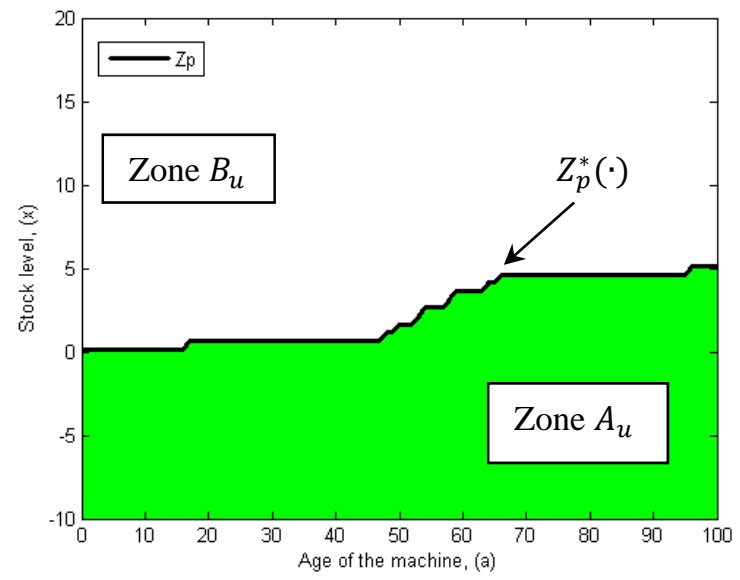

b) Production Trace $Z_{p}^{*}(\cdot)$

Figure 5: Optimal production policy

Examining Figures 5a and 5b, we see that the optimal stock level depends directly on the level of deterioration of the machine, denoted by its age $a(t)$. Consequently, the corresponding production policy is given by the following machine age-dependent hedging point policy:

$$
u(1, x, \mathrm{a})^{*}=\left\{\begin{array}{cc}
u_{\max } & \text { if } x(t)<Z_{p}^{*}(\cdot) \\
d & x(t)=Z_{p}^{*}(\cdot) \\
0 & x(t)>Z_{p}^{*}(\cdot)
\end{array}\right.
$$

where $Z_{p}^{*}(\cdot)$ is the function that defines the optimal production threshold at the operational mode as illustrated in Figure 5b. This production policy highlights the fact that the optimal stock level $Z_{p}^{*}(\cdot)$ is not fixed. In fact it changes depending on the deterioration of the machine. This means that the number of parts to hold in inventory to hedge against more frequent breakdowns and increasing defectives is adjusted to higher values as the deterioration of the machine increases. 


\subsection{Overhaul control policy}

Secondly, we shall discuss in this subsection the optimal overhaul policy obtained from the numerical example. From Figure 6a, we can observe that when the age of the machine is moderate, the overhaul activity is not recommended. This is because the rate of defectives $\beta$ and the transition $q_{12}$ remains in a rather small value with moderate ages, and the demand of products is not seriously affected. Conversely, when the age of the machine increases, the demand satisfaction of products begins to face serious problems, and then the overhaul activity is recommended to resolve the effects of deterioration. From the pattern of Figure 6a, we observe that the area to perform the overhaul activities spreads in the grid as a function of the age of the production system. To better illustrate the overhaul policy, we use its trace, presented in Figure 6b, where we notice that this policy divides the plane $(x, a)$ in two regions, such that the overhaul rate is set to its minimum or maximum value $\left(\omega_{\underline{o}}, \omega_{\bar{o}}\right)$ mainly according to the age of the machine. The description of these two zones is as follows:

- Zone $A_{o}$ : here, the age of the machine has reached such a level that the machine must be sent to overhaul activities, hence the decision variable $\omega_{o}(\cdot)$ is set to its maximum value.

- Zone $B_{o}$ : in this zone, the performance of an overhaul is not recommended, and the decision variable $\omega_{o}(\cdot)$ remains at its minimum value.

It should be noted that upon simultaneous consideration of the production and overhaul boundaries as presented in Figure 6b, only a part of the overhaul Zone $A_{o}$ is utilized, since the stock level is limited by the production threshold $Z_{p}^{*}(\cdot)$. This implies a reduction in the zone $A_{o}$ defining the feasible overhaul zone $A_{o}^{\prime}$.

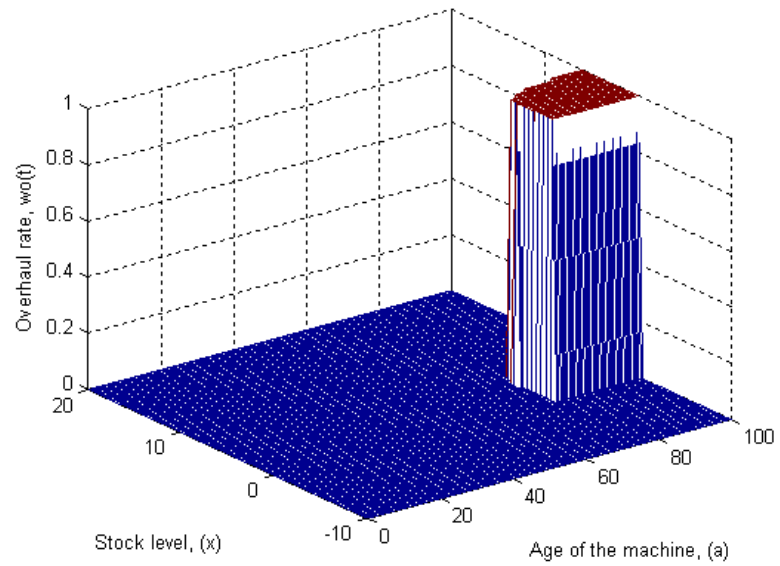

a)

Overhaul rate versus stock level and age of the machine

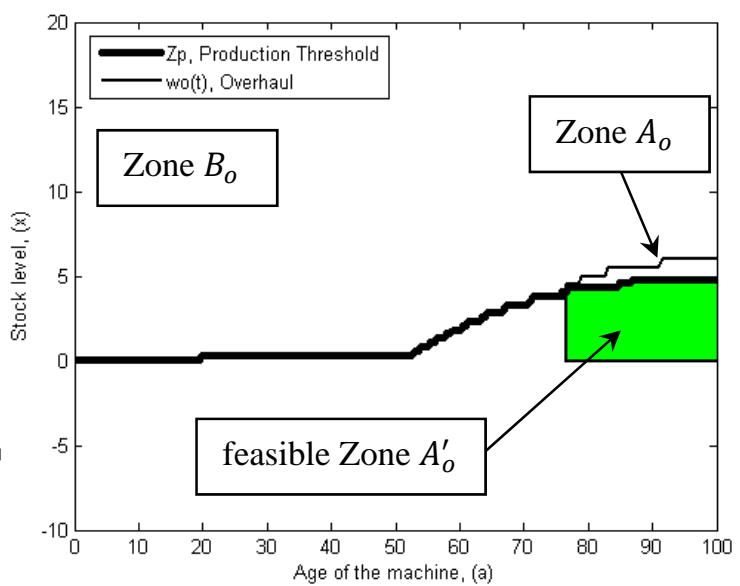

Figure 6: Overhaul policy 
The overhaul activity is triggered according to a machine age-dependent policy as presented in Figure 6b. This optimal policy states that overhaul activities must be performed at a rate $\omega_{o}^{*}(\cdot)$ given by the following equation:

$$
\omega_{o}^{*}(1, x, \mathrm{a})= \begin{cases}1 & \text { if } \mathrm{a}(t) \text { and } x(t) \in \text { zone } A_{o}^{\prime} \\ 0 & \text { otherwhise }\end{cases}
$$

These results may be described verbally by saying that the overhaul policy highlights the fact that as the age of the machine increases, its deterioration becomes worse, and this indicates that the machine must be sent to major overhaul to completely resolve the effects of deterioration. Clearly, the dependence of the age of the machine on the decisions involved indicates the influence of the deterioration on the overhaul policy.

\subsection{Preventive Maintenance Policy}

We terminate this section with the discussion of the preventive maintenance policy. From Figure $7 \mathrm{a}$, it is clear that the age of the machine has to reach a certain level to justify the cost of preventive maintenance. To facilitate the analysis of the preventive maintenance policy we use its boundary, as presented in Figure 7b. Such a boundary divides the plane $(\mathrm{x}, \mathrm{a})$ into two zones:

- Zone $A_{p}$ : it is recommended to send the machine to preventive maintenance, thus the decision variable $\omega_{p}(\cdot)$ is set to its maximum value.

- Zone $B_{p}$ : the policy consist in not conducting preventive maintenance, and so the decision variable $\omega_{p}(\cdot)$ is set to its minimum value.

A key point about the production threshold $Z_{p}^{*}(\cdot)$ is that it limits the stock level, and this defines the feasible preventive maintenance Zone $A_{p}^{\prime}$ as presented in Figure $7 \mathrm{~b}$, where we illustrate the trace of the joint production, overhaul and preventive maintenance policies. From the numerical results, we note that preventive maintenance is always conducted before overhaul, because preventive maintenance is less expensive and takes less time than the overhaul activities. The major overhaul is performed only when the deterioration of the machine has reached a much higher level that justifies its more expensive cost. 

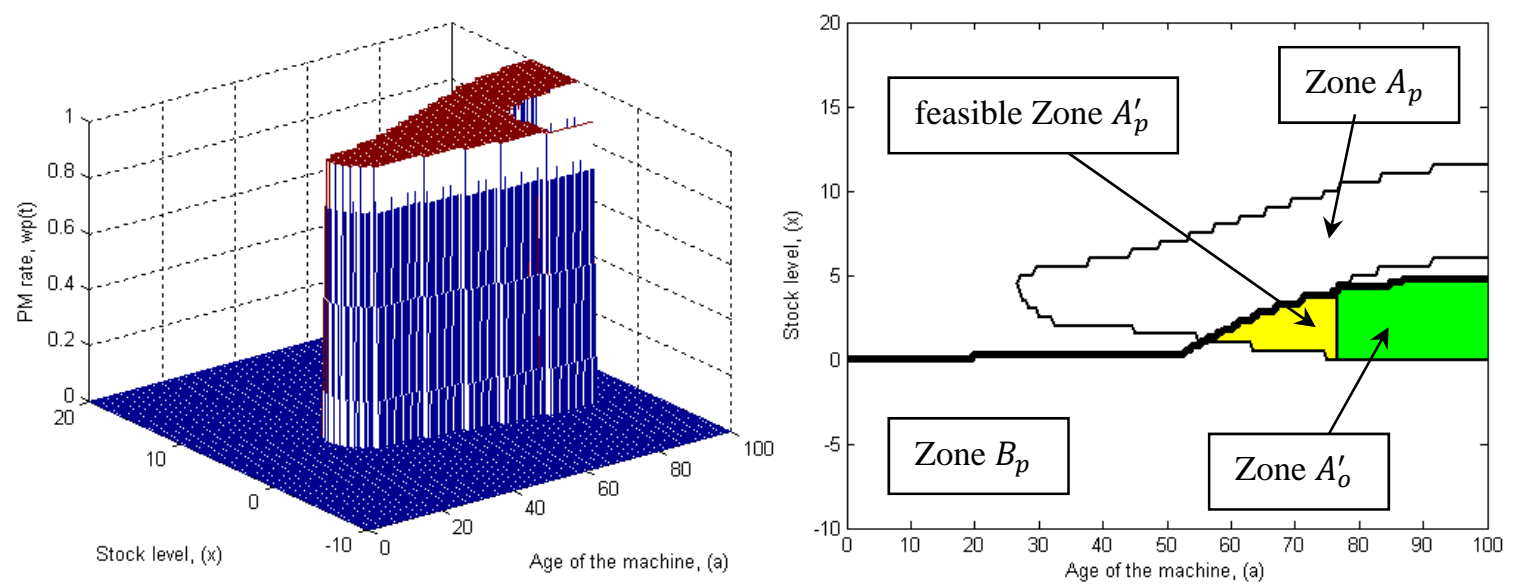

a) PM rate versus stock level and age of the machine

Figure 7: Preventive maintenance policy

Based on the above results, we claim that the optimal preventive maintenance policy follows a switching function based on the age of the machine $\mathrm{a}(t)$ for a given stock level $x(t)$ and it has a bang-bang structure, denoted as follows:

$$
\omega_{p}^{*}(1, \mathrm{a}, x)= \begin{cases}1 & \text { if } \mathrm{a}(t) \text { and } x(t) \in \text { zone } A_{p}^{\prime} \\ 0 & \text { otherwise }\end{cases}
$$

Bearing in mind the numerical results obtained so far, we can state that the simultaneous production, overhaul and preventive maintenance policy are perfectly defined by Equations (25)-(27) and implementing this control policy is possible to govern the manufacturing system by monitoring the stock level $x(t)$ and the deterioration of the machine, denoted by its age a $(t)$. The production policy can be completely parameterized by the control factor $Z_{p}^{*}(\cdot)$, meanwhile the overhaul and preventive maintenance policies can be defined by the zones $A_{o}^{\prime}$ and $A_{p}^{\prime}$, respectively. In order to confirm and validate the structure of the obtained optimal control policy, an extensive sensitivity analysis is performed in the next section. This analysis also will illustrate the usefulness of the obtained control policy.

\section{Sensitivity and results analysis}

The present section provides further evidence of the usefulness of the obtained control policy. We performed an extensive sensitivity analysis to illustrate the contribution of the joint policy, and also it permits to confirm its structure. The sensitivity analysis is conducted according to the variation of several parameters such as: the inventory cost, backlog cost, overhaul cost, preventive maintenance cost and defectives cost. Furthermore we analyze the effect of other parameters as well, including: the efficiency $\phi_{p}$ of the preventive maintenance and the adjustment parameters $\theta_{\mathrm{f}}$ and $\theta_{\mathrm{d}}$, related to the trend of the failure rate and the rate of defectives. 


\subsection{Variation of the inventory cost}

The sensitivity analysis begins with the discussion about the effect of the inventory cost. We present the production trace $Z_{p}^{*}$ in Figure 8a, for different inventory cost values $c^{+}=8,11$ and 14 . The results have been obtained by applying the numerical methods presented in the previous section. From Figure 8a we notice that when the inventory cost is moderate, i.e., $c^{+}=8$, the production threshold has the highest values of the analyzed cases and it starts increasing at around age $a=42$. When the inventory cost increases to $\mathrm{c}^{+}=11$, the optimal stock level follows a similar trend but below the previous case, in this scenario the production threshold begins its increase at age $a=47$. If we set the inventory cost to $c^{+}=14$, we observe a considerable reduction in the production threshold $Z_{p}$, since it begins its increase at around age $a=52$. From the results presented in Figure 8a, we can state that the more the inventory cost increases, the more the optimal stock level decreases. The reason for this condition is that with a higher inventory cost, the stock of the product is more penalized, hence reducing the production threshold. Conversely with lower inventory cost there is more liberty to maintain stock, and so the production threshold increases. Moreover, we note that the deterioration of the machine has a significant effect on the production policy, because the production threshold increases as the age of the machine increases. In particular, our results show that considerable ages denote higher rates of defectives and failures that are more frequent. Therefore, the production threshold increases as protection against the twofold effect of deterioration.

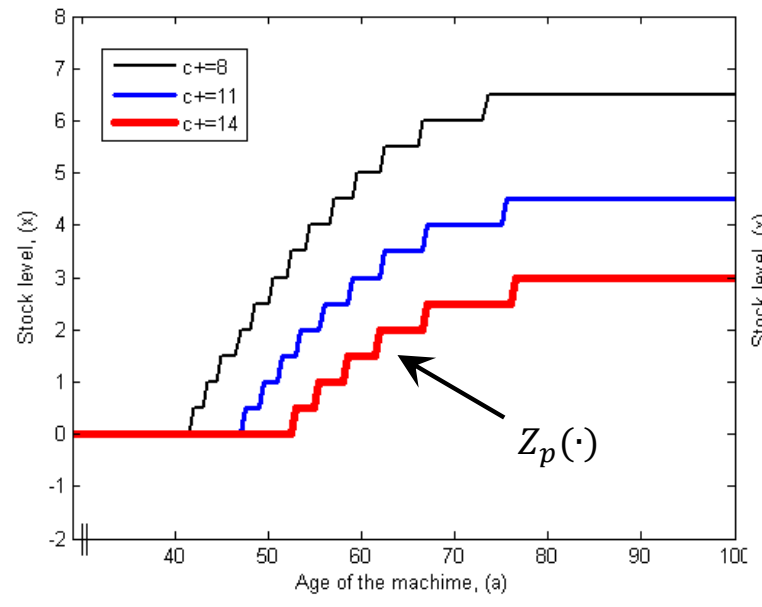

a) Effect on the production threshold $\mathrm{Z}_{\mathrm{p}}$

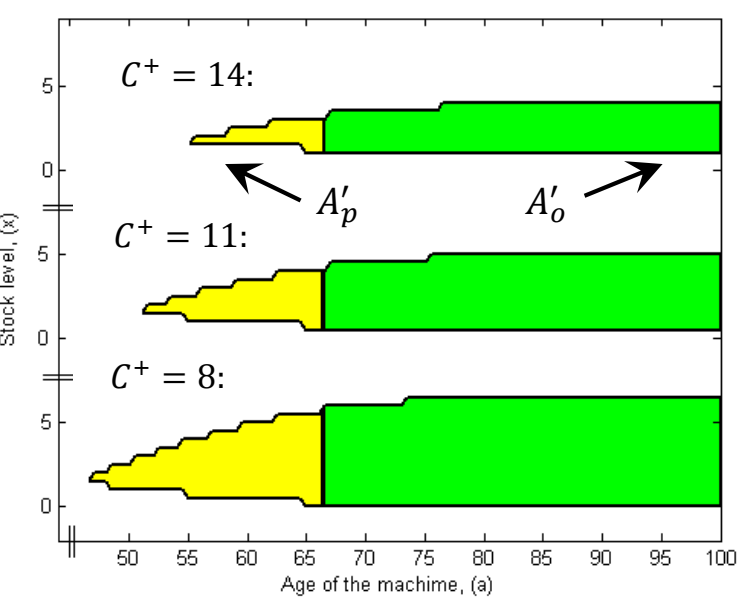

b) Effect on the zones $A_{p}^{\prime}$ and $A_{o}^{\prime}$

Figure 8: Sensitivity to the variation of the inventory cost

To complement the analysis of the inventory cost, we discuss its effect on the preventive maintenance and overhaul policies. We present in Figure $8 \mathrm{~b}$ three cases, using the same data cost as earlier, from them we observe that when the inventory cost is low, i.e., $\mathrm{c}^{+}=8$, the zone for preventive maintenance $A_{p}^{\prime}$ and the zone for overhaul $A_{o}^{\prime}$, cover a more extensive area in the computational domain. When the inventory cost increases to $c^{+}=11$, 
the zones $A_{p}^{\prime}$ and $A_{o}^{\prime}$ reduce, and they decrease even more when the inventory cost increases to $\mathrm{C}^{+}=14$. The reductions are explained because with higher inventory cost, the production threshold reduces, intersecting the zone $A_{p}$ and $A_{o}$ at a lower position, thus reducing the feasible zones $A_{p}^{\prime}$ and $A_{o}^{\prime}$. Recall that the production threshold is utilized to define the feasible zones for preventive maintenance and overhaul as mentioned previously in Figure 7b.

\subsection{Variation of the backlog cost}

The variation of the backlog cost $\mathrm{c}^{-}$, affects considerably the optimal production threshold as presented in Figure 9a, where the production trace for three different cost values $\mathrm{c}^{-}=110,150$ and 200 are analyzed. Analyzing the results of Figure 9a, we notice that when the backlog cost is $c^{-}=110$, the production thresholds are at their lowest levels for the entire analysis, and starts to increase at age $a=54$. If we increase the backlog cost to $c^{-}=150$, the production threshold also increases, but in this case it begins to increase considerably at age $a=47$. Additionally when $c^{-}=200$, the production threshold $Z_{p}$ increases even more than the previous cases, a notable increment from age $a=42$ is observed. Based on the analysis of these results, we can infer that the underlying pattern for the backlog cost implies that when the backlog cost increases, the production threshold also increases. This pattern suggests that since the backlog of product is more penalized with higher backlog cost, more products must be kept to protect the system from shortages and defectives. The increase in the stock level also indicates the effect of the deterioration in the production level, since to ensure demand satisfaction, the stock level increases when the age of the machine increases as well. Consequently higher ages indicate the presence of more disturbances due to more frequent breakdowns, and the presence of more defectives.

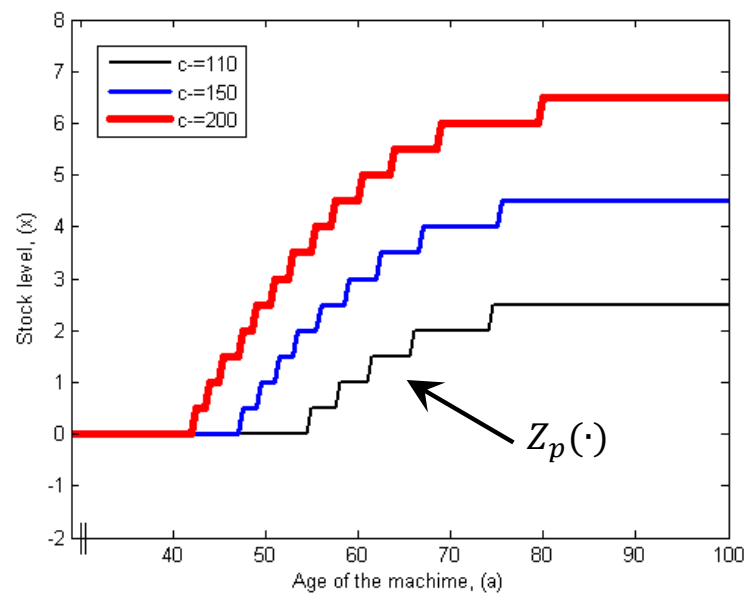

a) Effect on the production threshold $Z_{p}$

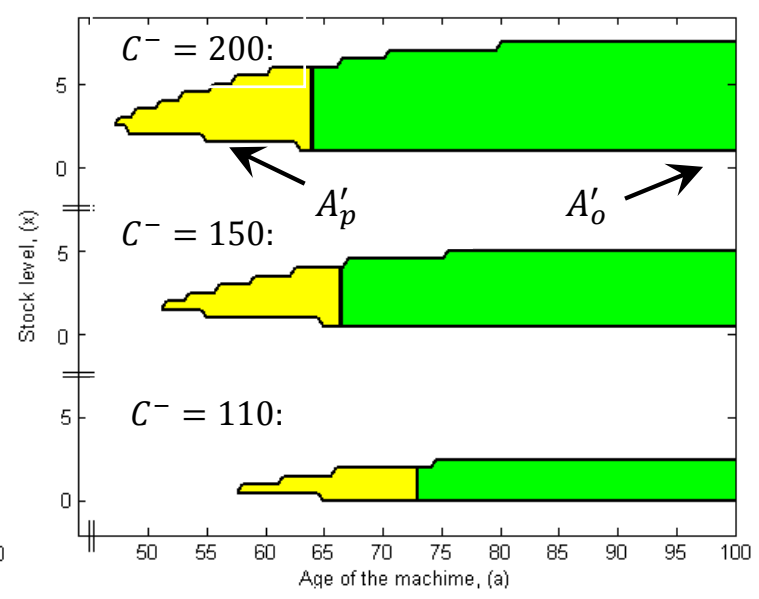

b) Effect on the zones $A_{p}^{\prime}$ and $A_{o}^{\prime}$ Figure 9: Sensitivity to the variation of the backlog cost 
We next turn our attention to the effect of the variation of the backlog cost on the preventive maintenance and overhaul policies. This variation is illustrated in Figure 9b, where we compare three cases. From these results, we observe that when the backlog cost is moderate, i.e., $\mathrm{c}^{-}=110$, preventive maintenance and overhaul are less recommended. When the backlog cost increases to $\mathrm{c}^{-}=150$, the zone for preventive maintenance $A_{p}^{\prime}$ and the zone for overhaul $A_{o}^{\prime}$ cover a larger area in the plane $(\mathrm{a}, x)$. In addition, if we increase the backlog cost to $\mathrm{c}^{-}=200$, both zones $A_{p}^{\prime}$ and $A_{o}^{\prime}$ increase even more. The intuition behind this pattern is that if the backlog cost increases, the production threshold increases as well, thus intersecting the zone $A_{p}$ of preventive maintenance and the zone $A_{o}$ of overhaul at a higher position, and this in consequence, increases the feasible zones $A_{p}^{\prime}$ and $A_{o}^{\prime}$. Thus, we can infer that the backlog cost is directly linked to the size of the zones of preventive maintenance and overhaul, since both zones increase according to the value of this cost. Besides, as expected from the results of Figure 8 and Figure 9, we observe that the effect of the backlog cost on the control policy is the inverse of the effect of the inventory cost.

\subsection{Variation of the preventive maintenance cost}

The results of three different cases are presented in Figure 10 to examine the variation of the preventive maintenance cost and its influence in the optimal policy. The analysis is made with the values $C_{p m}=2,4$ and 6 . From the numerical results, we observe that when the preventive maintenance cost is low, for instance $C_{p m}=2$, the preventive maintenance performance zone is the most extended of the analyzed cases. As the preventive maintenance cost increases to $C_{p m}=4$, the zone $A_{p}^{\prime}$ on the grid is reduced, and reduces even further with a higher cost of $C_{p m}=6$. From these results, we can deduce that the variation of the preventive maintenance cost mainly affects the preventive maintenance and overhaul policy as observed in Figure 10. Additionally this cost $C_{p m}$, has not reported any effect on the production policy, since the production threshold remained the same for the three analyzed cases. To an extent, the observed pattern implies that for a high preventive maintenance cost, the zone $A_{p}^{\prime}$ reduces considerably; indicating more overhauls, as should be logically expected. In addition, we notice that, since preventive maintenance is less expensive and takes less time, it is always conducted before major overhauls. Preventive maintenance is only carried out for an intermediate level of deterioration, when the age of the machine has reached a certain level to justify the cost of the activity, but if the age reaches a higher level of deterioration, it is more convenient to perform the overhaul instead. 


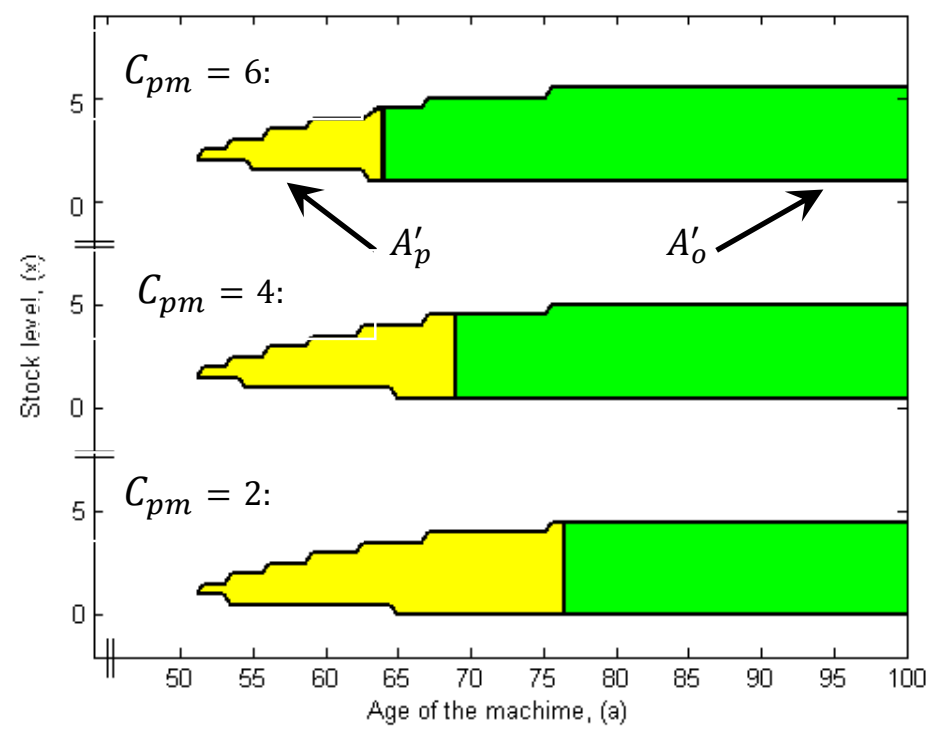

Figure 10: Sensitivity to the variation of the preventive maintenance cost and its effect on the preventive maintenance and overhaul policies

\subsection{Variation of the overhaul cost}

The sensitivity analysis of the overhaul cost is presented in Figure 11, and is performed based on the numerical results of three different cases with values defined as $C_{o}=10,12$ and 14. From the numerical results it follows that when the overhaul cost is low, i.e., $C_{o}=10$, we observe that the overhaul zone $A_{o}^{\prime}$ is the most prominent of the analyzed scenarios. When the overhaul cost increases to $C_{o}=12$, we notice a significant reduction in the zone $A_{o}^{\prime}$, therefore fewer overhauls are conducted. With a higher overhaul cost of $C_{o}=14$, the zone $A_{o}^{\prime}$ decreases more considerably. From Figure 11, the main issue is clearly that the variation of the overhaul cost has a significant effect on the preventive maintenance and overhaul policies, where we note upon increasing this $\operatorname{cost} C_{o}$, the zone $A_{o}^{\prime}$ is reduced, thus less overhauls are conducted. With respect to the production policy, we observe that the overhaul cost does not change the production threshold, since they remained unchanged for the three cases. Moreover, it is clear that as the major overhaul is more expensive and takes more time than the preventive maintenance, the overhaul is carried out only at a high level of deterioration, when the age of the machine is high enough to justify the higher cost of the overhaul. 


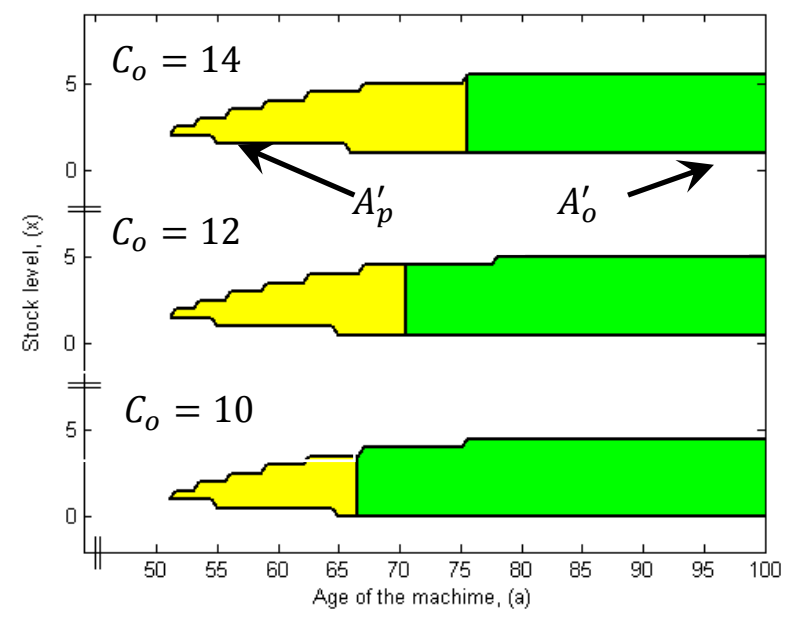

Figure 11: Sensitivity to the variation of the overhaul cost and its effect on the preventive maintenance and overhaul policies

\subsection{Variation of the defectives cost}

In this subsection, we highlight the preventive maintenance and overhaul policies under the effect of the variation of the defectives cost. Figure 12 indicates these polices with the values $C_{\mathrm{d}}=1,4$ and 7 . The size of the zone where preventive maintenance is recommended is the most considerable when $C_{\mathrm{d}}=1$. Nonetheless, it can be observed that increasing the defectives cost to $C_{\mathrm{d}}=4$, has a significant effect on these policies, reducing the preventive maintenance zone $A_{p}^{\prime}$. Furthermore, we note that preventive maintenance is less often initiated when the defectives cost increases to $C_{\mathrm{d}}=7$. These results show clearly that as the defectives cost increases, the preventive maintenance policy exhibits a pattern that recommends fewer numbers of this maintenance, and more overhauls. Additionally we note that this $\operatorname{cost} C_{\mathrm{d}}$ does not affect the production threshold. To make things clear, defective products are penalized more severely with higher defectives cost, and it follows that on increasing $C_{\mathrm{d}}$, the overhaul is more recommendable because it restores the level of deterioration of the machine more efficiently and rapidly. On conducting an overhaul, the rate of defectives and the MTTF are restored to AGAN conditions, whereas the imperfect preventive maintenance rejuvenates the machine only to a certain percentage. This logic explains the reasons why more overhaul should be performed as the defectives cost increases. 


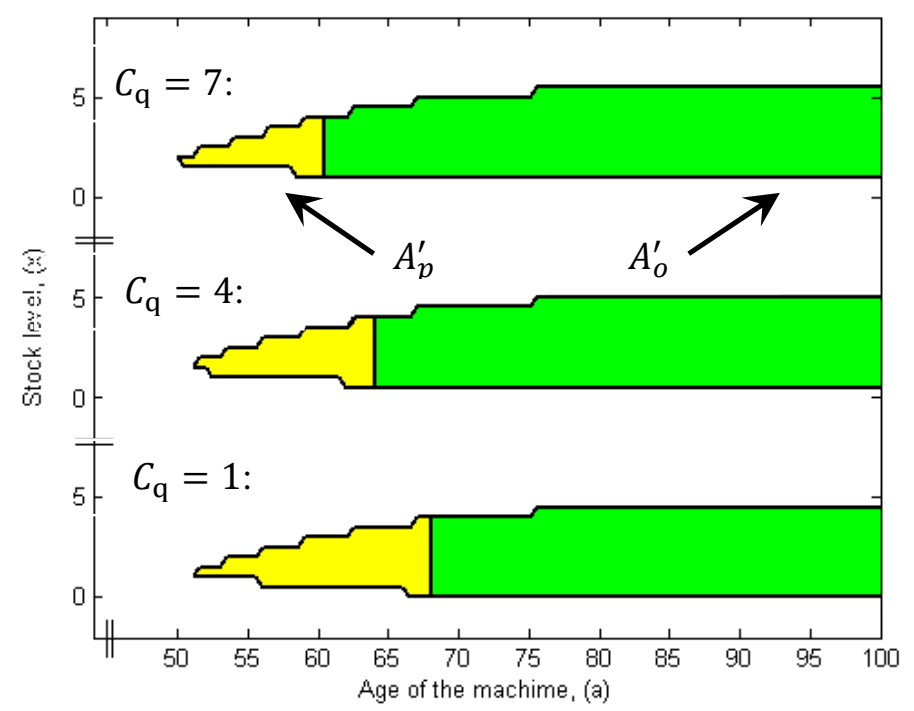

Figure 12: Sensitivity to the variation of the overhaul cost and its effect on the preventive maintenance and overhaul policies

\subsection{Variation of the preventive maintenance efficiency}

An equally significant parameter is the variation of the preventive maintenance efficiency $\phi_{p}$, since it has a remarkable effect mainly on the overhaul and preventive maintenance policies, as illustrated in Figure 13. The value of such efficiency $\phi_{p}$ has to be attractive enough to encourage preventive maintenance activities, otherwise this activity will not be recommended. This parameter $\phi_{p}$ is very useful since it enables the development of more realistic maintenance policies, modeling scenarios between the extreme cases of minimal and perfect repair, as discussed previously in section 2. In Figure 13, the variation of the preventive maintenance efficiency is presented, with values defined as; $\phi_{p}=0.55,0.6,0.65$ and 0.7. From these results we observe that when the efficiency $\phi_{p}$ is moderate, $\phi_{p}=0.55$, the zone for preventive maintenance $A_{p}^{\prime}$ is the least extensive on the plane $(\mathrm{a}, x)$. If we increase the efficiency to $\phi_{p}=0.6$, the zone $A_{p}^{\prime}$ increases, recommending more preventive maintenance, and when the efficiency further increases to $\phi_{p}=0.65$, the zone $A_{p}^{\prime}$ increases further. For the current numerical example, the results tell us that when the maintenance efficiency increases, the zone for preventive maintenance is significantly increased, because the incentive of conducting this activity is closer to the benefit of performing a perfect repair, reducing the level of deterioration to almost AGAN conditions. It can also be said that when the maintenance efficiency reaches a certain level, for example, $\phi_{p}=0.7$, preventive maintenance completely displaces the major overhaul because with this efficiency level and given the cost of both activities, it is a better option to conduct preventive maintenance. Conversely, it is no surprise to note that when maintenance efficiency decreases, the zone $A_{p}^{\prime}$ is considerably reduced, because the benefit of preventive maintenance becomes closer to ABAN conditions, and the reductions on the rate of defectives and MTTF are negligible. Therefore, the main point regarding the efficiency $\phi_{p}$ is that the difference in reduction on the age of the machine (and their corresponding change in the rate of defectives $\beta$ and 
MTTF), is the fundamental reason to explain the variations observed in the preventive maintenance zones of Figure 13. The effect of the efficiency $\phi_{p}$ is observed only on the preventive maintenance and overhaul policies, while the production thresholds remain the same for the four cases.

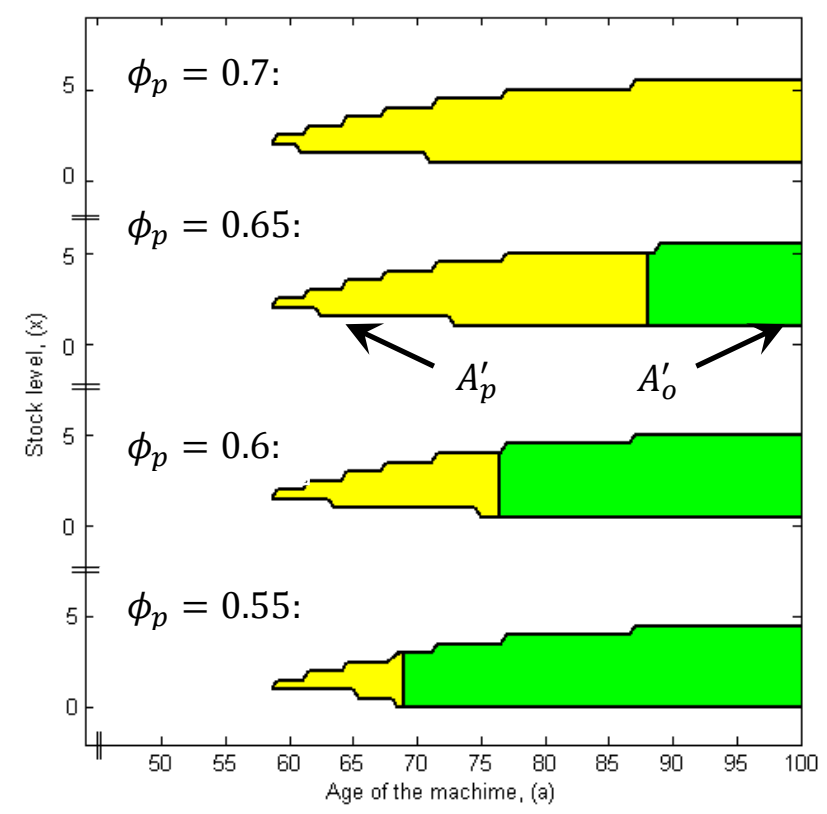

Figure 13: Sensitivity to the variation of the preventive maintenance efficiency $\phi_{p}$ and its effect on preventive maintenance and overhaul policies

\subsection{Variation of the adjustment parameter for the failure intensity}

In Figure 14a we illustrate the effect of the variation of the adjustment parameter $\theta_{\mathrm{f}}$ on the production policy. As discussed previously in section 2, this parameter $\theta_{\mathrm{f}}$ allows to adjust the trend of the failure intensity, denoted by the transition $q_{12}$. In Figure 14a the adjustment parameter takes three values $\theta_{\mathrm{f}}=0.4,0.6$ and 1 . When the parameter is low, $\theta_{\mathrm{f}}=0.4$, it means that the system experiences fewer failures, thus the production threshold $Z_{p}$ reduces. There are more frequent failures when $\theta_{\mathrm{f}}=0.6$, hence the production threshold increases as protection. If the parameter is set to $\theta_{\mathrm{f}}=1$, the machine needs more protection against the more frequent failures, leading to an even greater increase in the production threshold. It is worthwhile to mention that when the parameter $\theta_{\mathrm{f}}$ increases, the emphasis of deterioration is on more frequent failures, increasing more abruptly transition $q_{12}$. This affects the MTTF in such a way that reduces the reliability of the production system, promoting the increase of the production threshold as protection for shortages. 


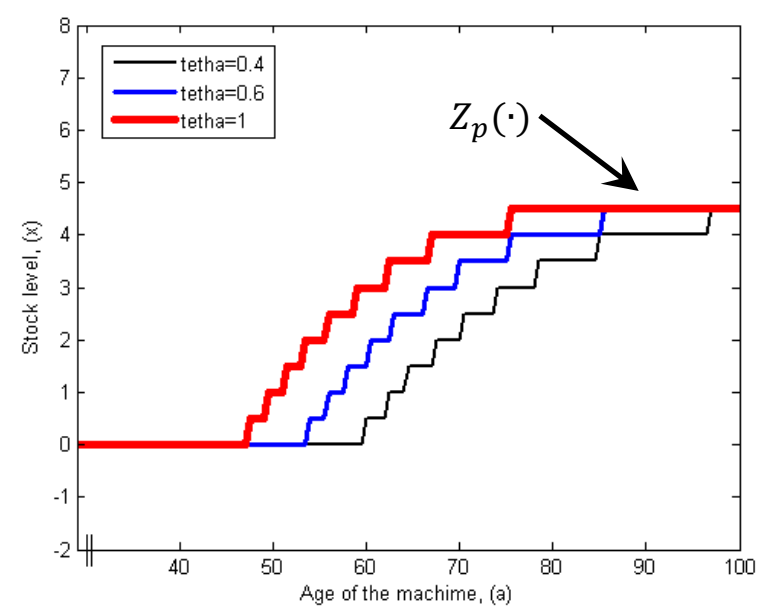

a) Effect on the production threshold $Z_{p}$

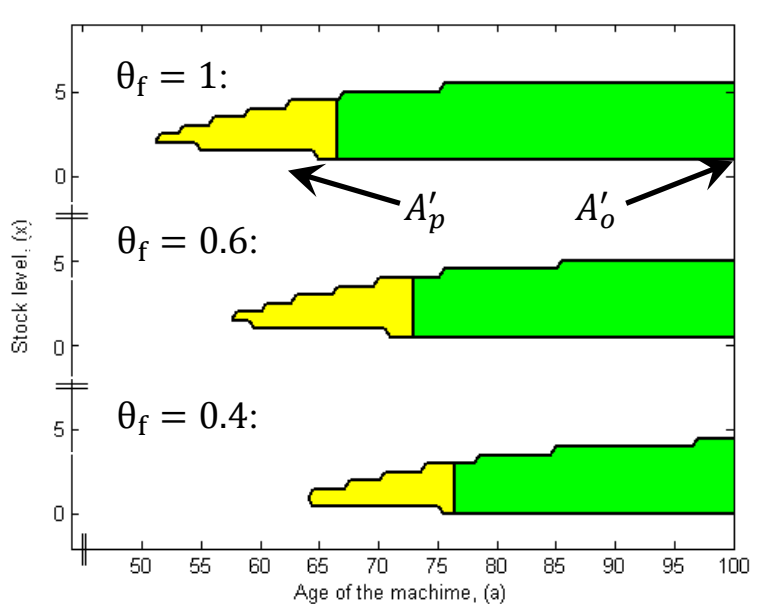

b) Effect on the zones $A_{p}^{\prime}$ and $A_{o}^{\prime}$

Figure 14: Sensitivity to the variation of the adjustment parameter $\theta_{f}$

As a matter of interest let us now analyze the effect of $\theta_{\mathrm{f}}$ on the preventive maintenance and overhaul policies. From the results of Figure 14b we notice that when the parameter is set to a low value such as $\theta_{\mathrm{f}}=0.4$, both the preventive maintenance zone $A_{p}^{\prime}$ and the overhaul zone $A_{o}^{\prime}$ are the smallest in the illustration. When the parameter increases to $\theta_{\mathrm{f}}=0.6$, both zones expand along the plane $(\mathrm{a}, x)$. And these zones continue their expansion when the parameter increases to $\theta_{\mathrm{f}}=1$. From this pattern we can draw a first inference that any variation in the production threshold $Z_{p}$ will certainly affect the total zone for preventive maintenance and overhaul, precisely because the zones $A_{p}^{\prime}$ and $A_{o}^{\prime}$ are delimited by their intersection with the stock level as presented previously in Figure $7 \mathrm{~b}$. A second observation is that with higher values of $\theta_{\mathrm{f}}$ we make more emphasis on failures rather than defectives, and from Figure 14b, it is clear that higher values of $\theta_{\mathrm{f}}$ encourage the conduction of more overhaul to more rapidly counter the effects of deterioration. It is not difficult to observe that there is a set of parameters with a strong influence on preventive maintenance and overhaul policies, with the potential to vary the obtained results. For example, looking at the maintenance efficiency employed in Figure 14b, (we used $\phi_{p}=0.6$ for the three cases), if we increase the efficiency to $\phi_{p}=0.65$, then we will observe a general reduction in the overhaul zone $A_{o}^{\prime}$, as reported in Figure 15a, because the preventive maintenance will be more attractive in such cases. However the trend is the same as in Figure 14b, promoting more overhaul as the value of $\theta_{\mathrm{f}}$ increases. This pattern is confirmed when we increase the overhaul cost from $\mathrm{c}_{\mathrm{o}}=10$ (in Figure 14b) to $c_{o}=14$, (in Figure 15b). With a higher $c_{o}$, the overhaul zone $A_{o}^{\prime}$ reduces considerably, and there appears then to be an interaction of several parameters that define the preventive maintenance and overhaul policies. Nevertheless a pattern is observed, the zone $A_{o}^{\prime}$ of overhaul increases, when the parameter $\theta_{\mathrm{f}}$ increases to cope more efficiently the presence of more frequent failures. 


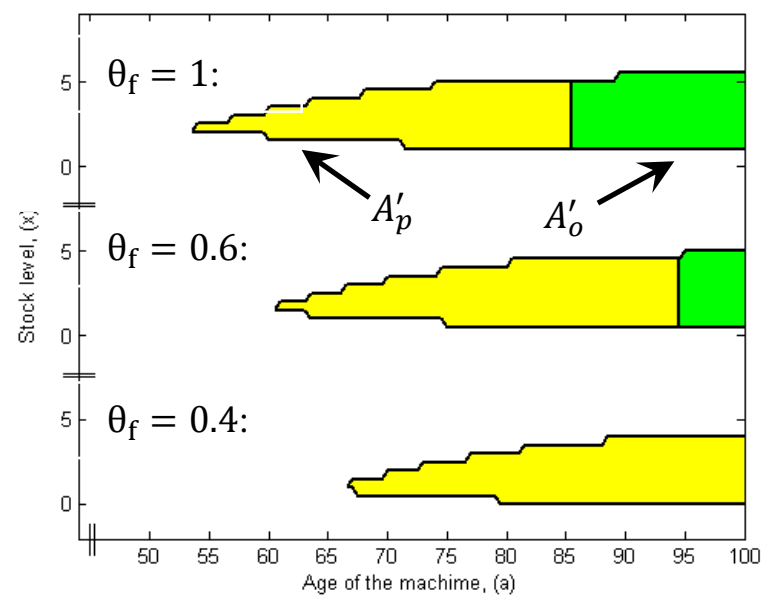

a) Zones $A_{p}^{\prime}$ and $A_{o}^{\prime}$ (with $\phi_{p}=0.65$ )

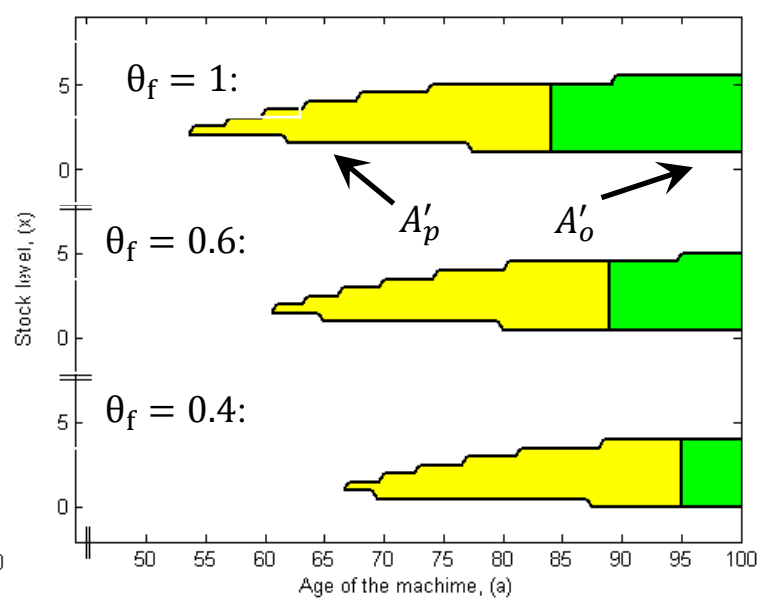

b) Zones $A_{p}^{\prime}$ and $A_{o}^{\prime}$ (with $\mathrm{c}_{\mathrm{o}}=14$ )

Figure 15: Sensitivity to the variation of the adjustment parameter $\theta_{\mathrm{f}}$

\subsection{Variation of the adjustment parameter of the rate of defectives}

In order to get an idea of the sensitivity of the adjustment parameter $\theta_{d}$ on the production control policy, we analyze three cases. Recall that this parameter $\theta_{\mathrm{d}}$ modifies the trend in the rate of defectives as a function of the age of the machine, as presented previously in Figure 3. There it can also be noted that when the age of the machine is significant enough, the rate of defectives will converge to the same limit value regardless of the amount of $\theta_{\mathrm{d}}$. We obtain the results presented in Figures 16a and Figure 16b, when the parameter $\theta_{\mathrm{d}}$ takes the values $\theta_{\mathrm{d}}=0.4,0.6$ and 1 . Whenever this parameter is set to a not-excessive value of $\theta_{\mathrm{d}}=0.4$, it means that the production threshold $Z_{p}$ does need to be so considerable because the deterioration of the machine does not imply a huge amount of defectives.

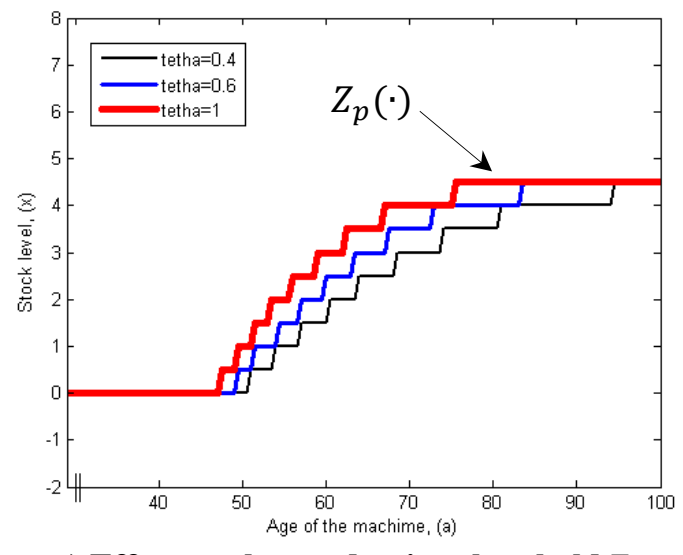

a) Effect on the production threshold $Z_{p}$

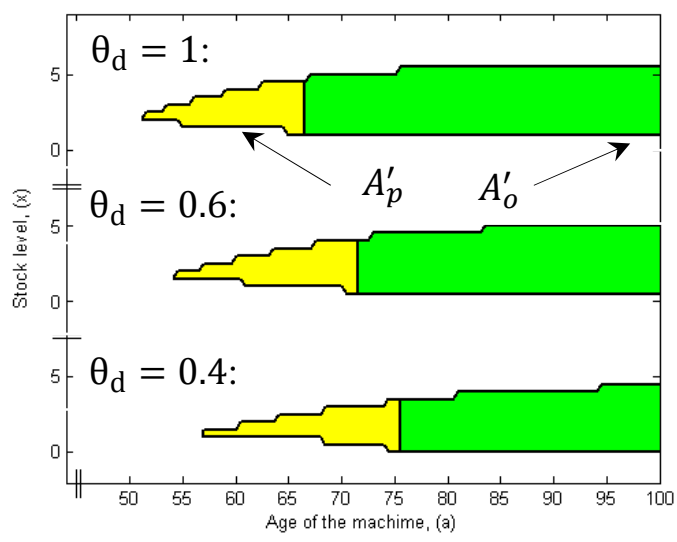

b) Effect on the zones $A_{p}^{\prime}$ and $A_{o}^{\prime}$

Figure 16: Sensitivity to the variation of the adjustment parameter $\boldsymbol{\theta}_{\mathrm{d}}$

Then, when $\theta_{d}=0.6$, the production threshold increases because the rate of defectives reaches higher values more rapidly. Additionally, if we increase the parameter to $\theta_{d}=1$, 
the rate of defectives increases so abruptly that in consequence the production threshold must increase further. On the basis of the results presented in Figure 16a, we can come to the observation that the role of the increase of the production threshold is to protect the system against the shortage of flawless products. When the parameter $\theta_{d}$ increases, the rate of defectives increases more abruptly, in consequence the demand of product increases as protection, and this causes an increment in the production threshold $Z_{p}$.

In essence, according to Figure $16 \mathrm{~b}$, the adjustment parameter $\theta_{\mathrm{d}}$ also has a clear effect on the preventive maintenance and overhaul policies. For instance, when $\theta_{\mathrm{d}}=0.4$, the zones in which preventive maintenance $A_{p}^{\prime}$ and overhaul $A_{o}^{\prime}$ are feasible are the smallest of the analyzed cases. On increasing the parameter to $\theta_{\mathrm{d}}=0.6$, the zone $A_{o}^{\prime}$ is more extensive on the grid. Moreover, when the parameter increases to $\theta_{\mathrm{d}}=1$, the zones $A_{o}^{\prime}$ expands further on the plane (a, $x$ ). As a consequence, we remark firstly, that any variation of the stock level modifies the total region of preventive maintenance and overhaul, denoted by the zones $A_{p}^{\prime}$ and $A_{o}^{\prime}$, since they are defined by their intersection with $Z_{p}$, as presented previously in Figure $7 \mathrm{~b}$. Secondly, the major difference in this case is that with higher levels of $\theta_{d}$, the emphasis is on more defectives instead of failures. More overhaul is conducted when the value of $\theta_{d}$ increases, because overhaul completely restores the rate of defectives with more efficiency than preventive maintenance. This pattern is confirmed, when we increase the preventive maintenance efficiency from $\phi_{p}=0.60$ (in Figure 16b) to $\phi_{p}=0.65$ (in Figure 17a), where we observe a similar pattern; the preventive maintenance zone $A_{p}^{\prime}$ decreases when $\theta_{\mathrm{d}}$ increases. Conversely, the overhaul zone $A_{o}^{\prime}$ grows when $\theta_{\mathrm{d}}$ increases. For closure, when we increase the overhaul cost from $c_{o}=10$ (in Figure $16 \mathrm{~b}$ ) to $c_{o}=14$, (in Figure 17b), the underlying pattern is the same, the overhaul zone $A_{o}^{\prime}$ increases when $\theta_{\mathrm{d}}$ increases, replacing the preventive maintenance zone $A_{p}^{\prime}$ that decreases.

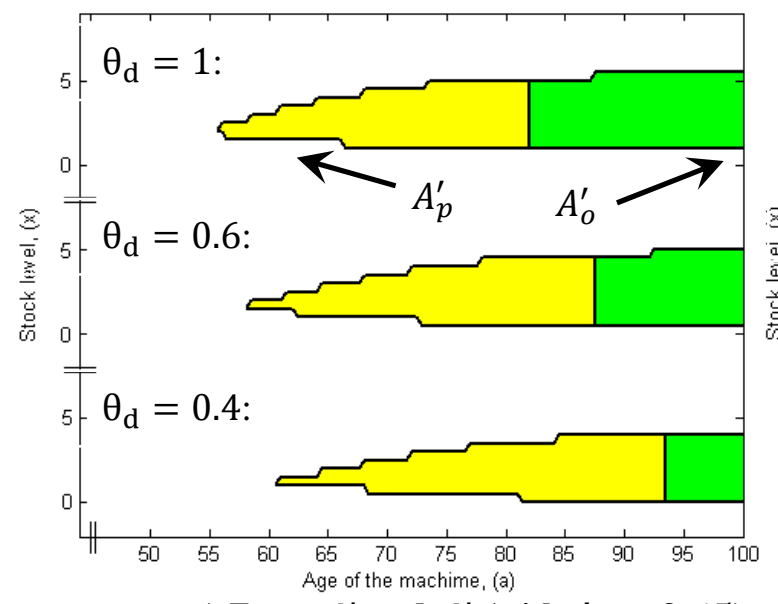

a) Zones $A_{p}^{\prime}$ and $A_{o}^{\prime}$ (with $\phi_{p}=0.65$ )

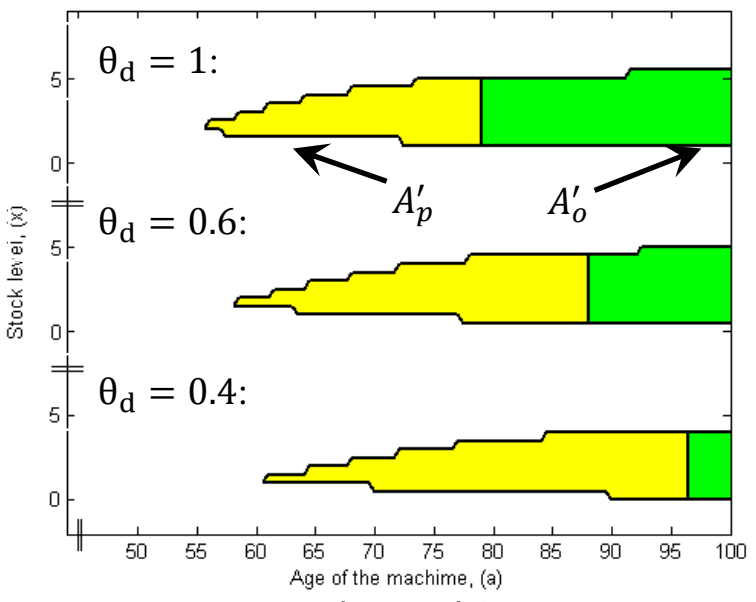

b) Zones $A_{p}^{\prime}$ and $A_{o}^{\prime}$ (with $\left.\mathrm{c}_{\mathrm{o}}=14\right)$

Figure 17: Sensitivity to the variation of the adjustment parameter $\boldsymbol{\theta}_{\mathrm{d}}$ 


\section{Discussions}

From what has been presented on the above sensitivity analysis, it is clearly enough to state that the simultaneous production, preventive maintenance and overhaul control policy is well characterized by the aforementioned control parameters $\left(Z_{p}, A_{o}^{\prime}\right.$ and $\left.A_{p}^{\prime}\right)$. For the manufacturing system considered, the joint control policy is given by Equations (25)-(27) and Figures 5 to 7. It is also important to note that the deterioration phenomenon influences the dynamics of several variables of the system, since on denoting deterioration with the age of the machine, its variation implies changes in the rate of defectives and the MTTF. As analysed in the above results with respect to the production policy, considering the effect of deterioration, it leads to a control policy that modifies the traditional hedging point policy to a situation with several threshold values that increase as a function of the age of the machine. Meanwhile, preventive maintenance and overhaul exhibit an age-dependent policy; they are triggered for a certain age level.

Through the sensitivity analysis conducted, we observe that the production threshold $Z_{p}$ decreases when the inventory cost increases, because the stock of the product is more penalized, and the contrary effect is observed for the backlog cost. There is also an increase in the production threshold $Z_{p}$ when the adjustment parameters $\theta_{\mathrm{f}}$ and $\theta_{\mathrm{d}}$ increase, this as protection against more frequent failures and the presence of more defective products. With regard to the preventive maintenance policy, when the preventive maintenance cost increases, the zone $A_{p}^{\prime}$ decreases, since this activity becomes more expensive. Furthermore, when we increase the preventive maintenance efficiency, there is a considerable increase in the zone $A_{p}^{\prime}$, because the incentive to promote this activity is more attractive, and on increasing the parameters $\theta_{\mathrm{f}}$ and $\theta_{\mathrm{d}}$ the zone $A_{p}^{\prime}$ decreases, since in such cases overhaul is more convenient. As we have seen in the previous sensitivity analysis, the overhaul zone $A_{o}^{\prime}$ reduces when the overhaul cost increases, and more overhauls are done when the defectives cost increases to completely cope the effects of deterioration. Further, when the parameters $\theta_{\mathrm{f}}$ and $\theta_{\mathrm{d}}$ increase, more overhaul is also performed. The relevance of the sensitivity analysis is apparent, since it seems that our results are logical and consistent, and this enables us to confirm the structure of the obtained joint control policy.

\section{Conclusion}

Is has been observed that the simultaneous production planning, preventive maintenance and overhaul control problem was addressed in this research work for the case of a single machine subject to random failures and deterioration. The main issue of the paper is to integrate quality aspects on the control policy, thus extending the concept of deterioration to create a connection with the rate of defectives and its reliability. We used a maintenance efficiency parameter to decrease the level of deterioration if preventive maintenance is carried out. We formulate a stochastic dynamic programming problem that integrates several types of maintenance; such as perfect, imperfect and minimal repair. Furthermore, on considering the machine's history, with the age of the production system, we devise a Semi-Markov decision model that enables us to take into 
account the twofold effect of deterioration: on the quality of the parts produced and the MTTF. Another important aspect is the fact that, our research analyzes the interaction of quality issues and the production control from the perspective of deteriorating systems. We illustrate the proposed approach thorough a numerical example, in which we observed that the stock level required as protection against failures and defectives increases as a function of the age of the production system. Meanwhile we found that the performance of preventive maintenance and overhaul activities depends on the stock level and the age of the machine. An extensive sensitivity analysis was also conducted. This serves to confirm the structure of the joint control policy. Finally, we conclude that the continuous deterioration of the machine has considerable effects on the joint production, preventive maintenance and overhaul policies, and the results obtained in this research are very satisfactory and encourage us to extend the study to more complex manufacturing systems.

\section{References}

[1] Akella, R., Kumar, P.R., 1986, Optimal control of production rate in a failure prone manufacturing system, IEEE Transactions on Automatic Control, vol. AC31, pp. 116-126.

[2] Boukas, E.K. and Hauire, A., 1990, Manufacturing flow control and preventive maintenance: a stochastic control approach, IEEE Transactions on Automatic Control 33, pp. 1024-1031.

[3] Colledani, M. and Tolio T., 2009, Performance evaluation of production systems monitored by statistical process control and off-line inspections. International Journal of Economics, 120, pp. 348-367.

[4] Colledani, M. and Tolio, T., 2011, Integrated analysis of quality and production logistics performance in manufacturing lines, International Journal of Production Research, 49:2, pp. 485-518.

[5] Chelbi A., Ait-Kadi D., 2004, Analysis of a production/inventory system with randomly failing production unit submitted to regular preventive maintenance. European Journal of Operational Research, n.156, pp. 712-718.

[6] Dehayem Nodem, F.I., Kenné, J.P., Gharbi, A., 2011, Production planning and repair/replacement switching policy for deteriorating manufacturing systems, International Journal of Advanced Manufacturing Technology, vol. 57, pp. 827840 .

[7] Dhouib, K., Gharbi, A., Ben Aziza, M.N., 2012, Joint optimal production control/preventive maintenance policy for imperfect process manufacturing cell, International Journal of Production Economics 137, pp. 126-136. 
[8] Doyen, L., and Gaudoin, O., 2004, Classes of imperfect repair models based on reduction of failure intensity or virtual age, Reliability Engineering \& System Safety, 84, pp. 45-46.

[9] Gharbi A., and Kenne, J.P., 2005, Maintenance scheduling and production control of multiple-machine manufacturing systems, Computer \& Industrial Engineering, vol.48, n. 4, pp. 693-707.

[10] Gharbi, A., Hajji, A., Dhouib, K., 2011, Production rate control of an unreliable manufacturing cell with adjustable capacity, International Journal of Production Research, 49:21, pp. 6539-6557.

[11] Gershwin, S.B., 2002, Manufacturing Systems Engineering, Massachusetts Institute of Technology, Second private printing, Cambridge, Massachusetts, USA.

[12] Hajji, A., Gharbi, A., Pellerin, R., 2012, Joint production control and product quality decision making in a failure prone multiple-product manufacturing system, International Journal of Production Research, 50:13, pp. 3661-3672.

[13] Inman R.R., Blumenfeld D.E., Huang, N., 2003, Designing production systems for quality: research opportunities from an automotive industry perspective, International Journal of Production Research, 41:9, pp. 1953-1971.

[14] Kenne, J.P. and Gharbi A., 1999, Experimental design in production and maintenance control problem of a single machine, single product manufacturing system, International Journal of Production Research, vol.37, n. 3, pp. 621-637.

[15] Kenné, J.P., Boukas, E.K., Gharbi, A., 2003, Control of Production and Corrective Maintenance Rates in a Multiple-Machine, Multiple-Product Manufacturing System. Mathematical and Computer Modeling, Pergamon, 38, pp. 351-365.

[16] Kim J., Gershwin S., 2008, Analysis of long flow lines with quality and operational failures, IIE Transactions, 40, pp. 284-296.

[17] Kim, J., Gershwin, S., 2005, Integrated quality and quantity modeling of a production line, OR Spectrum 27, pp. 287-314.

[18] Kimemia J., Gershwin S., 1983, An algorithm for the computer control of production in a flexible manufacturing system, 191-2216, 628-633 pp. IEEE.

[19] Kushner, H.J. and Dupuis, P.G., 1992, Numerical Methods for Stochastic Control Problems in Continuous Time, (Springer, New York, NY).

[20] Lai, M.T., and Chen, Y.C., 2006, Optimal periodic replacement policy for a twounit system with failure rate interaction, International Journal of Advanced Manufacturing Technology, 29 pp. 367-371.

[21] Lam, Y., Zhu, L.X., Chan, J.S.K., Liu, Q., 2004, Analysis of data from a series of 
events by a geometric process model, Acta Mathematicae Applicatae 20, pp. 263282.

[22] Love, C.E., Zhang, Z.G., Zitron, M.A., Guo, R., 2000, A discrete semi-Markov decision model to determine the optimal repair/replacement policy under general repairs, European Journal of Operational Research, 125 pp. 398-409.

[23] Mhada, F., Hajji, A., Malhame, R., Gharbi, A., Pellerin, R., 2011, Production control of unreliable manufacturing systems producing defective items, Journal of Quality in Maintenance Engineering, Vol. 17, No. 3, pp. 238-253.

[24] Mok, P.Y., Porter, B., 2005, Evolutionary optimization of hedging points for unreliable manufacturing systems, International Journal of Advanced Manufacturing Technology, 28 pp. 205-214.

[25] Radhoui, M., Rezg, N., Chelbi, A., 2010, Integrated maintenance and control policy based on quality control, Computers \& Industrial Engineering, Vol. 48, pp. 443-451.

[26] Rivera-Gomez, H., Gharbi, A., Kenné, J.P., 2013, Production and quality control policies for deteriorating manufacturing system, International Journal of Production Research, accepted paper.

[27] Sajadi, S.M., Seyed Esfahani M.M., Sörensen, K., 2011, Production control in a failure-prone manufacturing network using discrete event simulation and automated response surface methodology, International Journal of Advanced Manufacturing Technology, 53 pp. 35-46.

[28] Tempelmeier, H., Bürger, M., 2001, Performance evaluation of unbalanced flow lines with general distributed processing times, failures and imperfect production. IIE Transactions 33, pp. 293-302.

[29] Wu, S. and Clements-Croome, D., 2006, A novel repair model for imperfect maintenance. IMA Journal of Management Mathematics, 17, pp. 235-243.

[30] Yulan, J., Zuhua, J., Wenrui, H., 2008, Multi-objective integrated optimization research on preventive maintenance planning and production scheduling for a single machine, International Journal of Advanced Manufacturing Technology, 39 pp. 954-964. 